\title{
Le marché des " subsistances ". L'exemple de la plaine de Montréal au début des années 1830 : une perspective géographique
}

\section{Serge Courville}

Volume 42, numéro 2, automne 1988

URI : https://id.erudit.org/iderudit/304678ar

DOI : https://doi.org/10.7202/304678ar

Aller au sommaire du numéro

Éditeur(s)

Institut d'histoire de l'Amérique française

ISSN

0035-2357 (imprimé)

1492-1383 (numérique)

Découvrir la revue

Citer cet article

Courville, S. (1988). Le marché des « subsistances ». L’exemple de la plaine de Montréal au début des années 1830 : une perspective géographique. Revue d'histoire de l'Amérique française, 42(2), 193-239.

https://doi.org/10.7202/304678ar
Résumé de l'article

La prolifération de villages et d'industries rurales observée dans les seigneuries du Québec au cours de la première moitié du $19^{\mathrm{e}}$ siècle attire l'attention des chercheurs sur le rôle qu'a pu jouer ce facteur dans les transformations de l'agriculture. Observé pour 1831, le phénomène semble avoir été déterminant, du moins dans la région de Montréal où la cartographie des données de recensement montre diverses symbioses entre agriculture, villages et industries rurales. Elle révèle aussi des contrastes très marqués entre les différents secteurs du territoire, qu'accentue la structuration en enveloppes de l'espace agricole. Loin d'être une source de tensions à l'intérieur de la région, ces contrastes semblent au contraire l'unifier, en favorisant une vie de relation dont témoignent la densité du réseau routier, la mobilité de la main-d'oeuvre, l'aire d'extension de certaines cultures ou de certains élevages autour des lieux de répartition des villages et des industries rurales, et le rôle que semblent jouer les entreprises locales dans la vocation de certains terroirs, même quand ceux-ci sont situés à distance des lieux immédiats de localisation des moulins ou des fabriques. En 1831, le paysage rural régional présente des traits qui rappellent ceux de certains pays européens où la montée de l'âge industriel fut préparée par une phase préalable de proto-industrialisation.
Tous droits réservés (C Institut d'histoire de l'Amérique française, 1988
Ce document est protégé par la loi sur le droit d'auteur. L’utilisation des services d'Érudit (y compris la reproduction) est assujettie à sa politique d'utilisation que vous pouvez consulter en ligne. 


\title{
LE MARCHÉ DES «SUBSISTANCES». L'EXEMPLE DE LA PLAINE DE MONTRÉAL AU DÉBUT DES ANNÉES 1830: UNE PERSPECTIVE GÉOGRAPHIQUE ${ }^{1}$
}

\author{
SERGE COURVILLE \\ Département de géographie \\ Université Laval
}

\begin{abstract}
RÉSUMÉ
La prolifération de villages et d'industries rurales observée dans les seigneuries du Québec au cours de la première moitié du 19e siècle attire l'attention des chercheurs sur le rôle qu'a pu jouer ce facteur dans les transformations de l'agriculture. Observé pour 1831, le phénomène semble avoir été déterminant, du moins dans la région de Montréal où la cartographie des données de recensement montre diverses symbioses entre agriculture, villages et industries rurales. Elle révèle aussi des contrastes très marqués entre les différents secteurs du territoire, qu'accentue la structuration en enveloppes de l'espace agricole. Loin d'être une source de tensions à l'intérieur de la région, ces contrastes semblent au contraire l'unifier, en favorisant une vie de relation dont témoignent la densité du réseau routier, la mobilité de la main-d'oeuvre, l'aire d'extension de certaines cultures ou de certains élevages autour des lieux de répartition des villages et des industries rurales, et le rôle que semblent jouer les entreprises locales dans la vocation de certains terroirs, même quand ceux-ci sont situés à distance des lieux immédiats de localisation des moulins ou des fabriques. En 1831, le paysage rural régional présente des traits qui rappellent ceux de certains pays européens où la montée de l'âge industriel fut préparée par une phase préalable de proto-industrialisation.
\end{abstract}

\begin{abstract}
The observance, within the first half of the nineteenth century, of the proliferation of villages and rural industries in Quebec's seigneuries, draws the attention of researchers to this phenomenon and to the role it might have played in the transformations of agriculture. This phenomenon reflected in the census data for the Montreal region in 1831, shows varied symbiotic relationships between agriculture, the villages and the rural industries. These relationships reveal marked contrasts, between the different parts of the territory, which are emphasized by the singular structuralizations in envelopes of the agricultural area. These contrasts, far from being a source

1 Ce texte est une version révisée d'une communication présentée au Séminaire d'histoire économique du département d'histoire de l'Université de Genève (mai 1988) et au congrès de l'Institut d'histoire de l'Amérique française (octobre 1988). Il résulte d'une recherche financée par le Fonds FCAR (Québec), à laquelle était associé Normand Séguin, du Centre de recherches en études québécoises (UQTR). Il doit beaucoup au travail de mes étudiants qui, pendant deux ans, ont patiemment dépouillé les microfilms de recensement. Parmi eux, Maryse Poudrier, Johanne Lachance, Nancy Gagné et André Crochetière, auxquels sont venus se joindre pendant un temps d'autres collaborateurs dont Jocelyn Morneau (UQTR). Il doit beaucoup, aussi, à Philippe Désaulniers, sur qui j'ai pu compter pour la mise en forme de mes fichiers informatiques et la plupart de mes travaux de cartographie assistée par ordinateur, ainsi qu'à l'équipe du laboratoire de cartographie de l'Université Laval qui a parachevé certaines de mes cartes.
\end{abstract}


of tension within the region, seems on the contrary to be a unifying force, favoring a life of relation, as indicated by the network of roads, the mobility of the labor force and the expansion of certain crops and livestock near the villages and rural industries. Indicated also is the role seemingly played by the local industries in the agricultural vocation, even when these industries were situated some distance from the mills and factories. The rural picture of 1831 resembles certain traits found in some european countries, where the advent of the industrial age was first preceeded by a proto-industrial phase.

L'un des plus importants débats de l'historiographie canadienne a tourné autour de l'agriculture au Bas-Canada. Ce débat est connu: d'un côté, il y a ceux qui l'ont vue comme une activité de subsistance, mal intégrée au marché et en butte à des difficultés de croissance qui auraient entraîné l'abandon du blé comme culture dominante; de l'autre, ceux qui l'ont vue comme une activité primitive mais rationnelle, capable de répondre positivement à la demande. Le construit scientifique a suivi, logique dans les deux cas, laissant voir deux types d'agriculture, l'une routinière, attachée au sol et aux valeurs familiales, et l'autre plus sensible au marché et mieux accordée aux transformations de l'appareil économique global. Toutefois, en dépit des écarts qui séparent les thèses, une image centrale demeure, celle d'une activité de subsistance, très proche encore de ses bases ancestrales, et dont les performances face au marché restent bien en deçà des progrès enregistrés ailleurs dans d'autres contrées du monde.

Aujourd'hui, les choses en sont là. Tout en souscrivant à l'idée de malaises manifestes surtout dans les années 1830, on accepte aussi celle d'une montée de l'économie de marché capable d'influencer l'agriculture. Pourtant, malgré des travaux aussi nombreux que féconds, plus sensibles qu'auparavant aux particularismes locaux, le problème reste entier, car, si les données s'accumulent, on manque encore de bases sûres de traitement; en outre, on connaît mal la géographie ancienne des campagnes; enfin, on ne dispose que de très peu d'études éclairant les organisations concrètes de l'agriculture à l'échelle régionale. Il devient donc difficile dans ce contexte de valider les enquêtes menées à l'échelle locale et d'en intégrer les bilans dans des synthèses, même provisoires, qui pourraient donner une nouvelle vision de l'agriculture.

Cette présentation se veut un propos d'étape dans nos travaux de reconstitution des paysages ruraux de la vallée du Saint-Laurent au cours de la première moitié du $19 \mathrm{e}$ siècle. Elle a pour thème le marché que semble avoir favorisé la montée de villages et d'industries rurales dans ce qui constituait alors le coeur économique du Bas-Canada, la région de Montréal (appelée aussi «district» de Montréal dans les sources), et qui s'organise autour de denrées et de produits dont on a souvent dit qu'ils ne servaient que des fins domestiques. Ce territoire, dont nous limiterons l'observation aux seules seigneuries et au début 
des années 1830 , n'est qu'à demi représentatif des autres régions du Bas-Canada, dont il se distingue à la fois par le relief, le climat et les sols. Toutefois, comme il réunit plus de la moitié de la population qui vit alors dans la vallée du Saint-Laurent et qu'il fut le site de troubles politiques importants, que plusieurs auteurs attribuent aux malaises croissants de l'agriculture, il paraît plus susceptible que d'autres d'éclairer le contexte dans lequel cette activité évolue.

\section{LE PRUBLÈME}

Dans leur évaluation de l'agriculture au Bas-Canada, les chercheurs ont surtout insisté sur la place du blé dans le système de cultures, faisant de sa récolte et du volume de ses exportations un reflet de la santé économique des campagnes et, partant, du dynamisme de la société rurale tout entière. Importante, cette question ne doit pourtant pas faire oublier le rôle qu'ont pu jouer l'élevage et les autres cultures dans l'économie rurale. Car si les transformations de la ferme entre la fin du $18 \mathrm{e}$ siècle et le début des années 1830 peuvent traduire une détérioration des conditions de l'agriculture, elles peuvent aussi refléter bien autre chose, par exemple la diversification de la demande sur le marché intérieur. Autrement dit, dans quelle mesure la ferme vit-elle coupée de son contexte? Se peut-il qu'elle ne subisse d'influences ni du village ni des industries rurales, pourtant en plein essor à l'époque?

Il ne s'agit pas de nier ici les difficultés qui confrontent l'agriculture au Bas-Canada, celles-ci sont nombreuses: fortes augmentations de population qui accroissent le nombre de bouches à nourrir, accidents climatiques, épidémies, prélèvements divers qui en compromettent la croissance, etc. Il s'agit plutôt de se demander si des cultures comme l'orge, le seigle, les pommes de terre, le tabac, ou si un élevage tel celui du mouton, n'ont servi que des fins d'autosuffisance, sans répondre également à la demande introduite par les brasseries, les distilleries, les manufactures à tabac, les tanneries, les moulins à fouler ou à carder rencontrés en milieu rural et dont le nombre est de plus en plus imposant. Et que dire des productions de laine et d'étoffe enregistrées après 1831: se peut-il qu'elles n'aient eu aucun précédent ou qu'elles ne témoignent que d'activités domestiques sans rapport avec l'industrie naissante du textile?

À ces questions, nous ne pouvons apporter pour l'instant qu'une réponse, celle des rapports qui paraissent s'être établis dans l'espace entre population, villages, agriculture et industries rurales, ceux-ci étant vus à partir d'une cartographie à grande échelle des données de recensement ${ }^{2}$. C'est peu sans doute pour résoudre le problème mais

\footnotetext{
2 Comme il s'agissait d'étudier ici une activité dont les contextes ont été très divers, il fallait pouvoir l'appréhender le plus précisément possible, c'est-à-dire en tenant compte à la fois
} 
suffisant pour le poser, car l'agriculture à l'époque n'est pas stimulée que par les grands marchés, elle l'est également par les marchés locaux et régionaux. Qui veut comprendre les organisations de cette activité dans l'espace et saisir les dynamismes qui la sous-tendent, doit aussi prendre en compte ces marchés, l'échelle d'analyse devenant ici celle de la territorialité du groupe humain observé, pour qui le marché des subsistances s'inscrit dans la logique d'un système d'échange que l'on aurait tort de limiter au grand marché international. Notre essai a pour but de montrer l'importance de ce marché et ce qui en découle dans l'organisation du territoire agricole. Il a aussi pour but de montrer en quoi l'étude des paysages peut contribuer à une meilleure saisie de l'économie et de la société; car à travers les formes et les fonctions de l'espace organisé, c'est tout une vie de relation qui se profile, dans ses expressions les plus structurantes.

\section{LE TERRITOIRE D'OBSERVATION, SES CARACTÉRISTIQUES}

Ce que nous appelons la région de Montréal correspond en gros à la plaine de Montréal, avec ses prolongements sous forme de vallées ou de bandes riveraines de basses terres, jusque dans les plateaux voisins. Elle couvre à peu près tout l'ouest du Bas-Canada, depuis l'entrée du lac Saint-Pierre à l'est jusqu'à la frontière avec le Haut-Canada à l'ouest, et s'étend des Laurentides au nord jusqu'aux Appalaches et à la frontière avec les États-Unis au sud. À lui seul, ce territoire comprend un peu plus de 80 fiefs et seigneuries et cumule environ trois millions d'arpents cultivables sur les huit millions que compte l'ensemble du territoire seigneurial ${ }^{3}$.

\footnotetext{
du lieu de sa pratique dans l'espace et de la distance de celui-ci au marché. Toutes les données ont été géocodées et cartographiées à différentes échelles. La première fut celle de la côte dont le dessin sur les cartes correspond aux tracés des cartes actuelles de cadastre, sauf dans le cas des côtes regroupées dans les mentions du recensement et dans celui des côtes que nous n'avons pu localiser, faute d'informations suffisantes dans les sources documentaires de la période (une douzaine sont dans ce cas, pour lesquelles il a fallu nous satisfaire de données moyennes cartographiées dans les secteurs probables de localisation de ces côtes). La deuxième échelle fut celle de la localité, c'est-à-dire de la subdivision de recensement, dont le dessin a pu être reconstitué à partir de travaux antérieurs. La troisième, celle de la sous-région (grand ensemble), à l'intérieur de laquelle nous avons distingué divers sous-ensembles (groupes de localités). Ont été retenus comme grands ensembles, l'archipel de Montréal, la "péninsule» Vaudreuil-Soulanges, et les rives nord et sud du fleuve; comme sous-ensembles, les secteurs riverains, ceux de l'intérieur, ceux de l'arrière-pays seigneurial, et celui de la vallée du Richelieu, laquelle a aussi été partagée en trois sous-ensembles: le Bas, le Moyen et le Haut-Richelieu. Quant à la cartographie ellemême, elle a été effectuée par informatique, sur micro-ordinateur MacIntosh pour les cartes à petite échelle, et grâce au programme Théma développé à l'Université Laval pour les cartes à moyenne et à grande échelles. Ce programme fonctionne sur système MUSIC et sur ordinateur IBM 4381.

${ }_{3}$ Maurice Séguin, La nation canadienne et l'agriculture 1760-1850 (Trois-Rivières, Le Boréal Express, 1970), 171-172.
} 


\section{Le milieu physique}

$\mathrm{Vu}$ de haut, cet espace prend la forme d'une large cuvette déprimée au centre, perçée çà et là de masses magmatiques et partagée en deux par un fleuve aussi puissant que large, divisé en trois bras principaux à la hauteur de l'archipel de Montréal, et auquel aboutit une multitude de cours d'eau, dont deux affluents importants, la rivière Richelieu sur la rive sud et la rivière des Outaouais au nord-ouest. Profondément marqué par la dernière grande glaciation et l'intrusion marine qui l'a suivie, cet espace présente des visages très divers que tendent à uniformiser les nappes d'argile et d'alluvions récentes, mais que singularisent par endroits des terrasses aux pentes parfois abruptes (les «Grands-Côteaux»), des dépôts de till glaciaire, des affleurements de moraine, des langues de sable et de gravier, et des tourbières. Ces contrastes caractérisent surtout la rive nord du fleuve où les conditions physiques du milieu sont très variées. Mais on les retrouve aussi dans l'archipel de Montréal, dans la «péninsule» Vaudreuil-Soulanges et sur la rive sud du fleuve où ils sont toutefois moins accentués, sauf peutêtre au nord-est où les placages de sable sont nombreux (secteurs de Sorel et de Saint-Ours), et au sud-ouest où les tourbières dominent ${ }^{4}$. Quant au climat, il est partout favorable, mais davantage sur la rive sud du fleuve, où la saison végétative a tendance à se prolonger.

\section{La population}

Le peuplement régional est ancien et remonte au début des années 1640 avec l'établissement de Ville-Marie dans l'île principale de l'archipel d'Hochelaga. De cette date jusqu'aux années 1760-1780, l'accroissement démographique est surtout alimenté par des courants migratoires venus de l'est, de la région de Québec notamment, qui lui a fourni ses plus forts contingents. Par la suite toutefois, et en dépit d'un apport extra-régional encore important, on assiste à un croît sur place des effectifs démographiques locaux auxquels viennent s'ajouter, dès la fin du $18 \mathrm{e}$ siècle et les premières décennies du $19 \mathrm{e}$, quelques milliers d'immigrants venus d'Europe ou des États-Unis. On les retrouve partout, à la ville notamment, mais aussi à la campagne où ils forment des communautés parfois imposantes. Les uns, plus pauvres, sont établis sur la frange du bouclier laurentien où ils recherchent surtout le travail en forêt; les autres, mieux nantis, sont installés sur de meilleurs sols, sur la rive sud du fleuve ou, comme les Loyalistes, à la frontière canado-américaine, où ils sont à l'origine de paysages spécifiques. Mais

\footnotetext{
4 Différents outils peuvent permettre une appréciation du milieu physique. Bien peu toutefois en donnent une vision d'ensemble. On en trouvera un exemple dans le bulletin technique no 4 du Service d'urbanisme de la Ville de Montréal, réalisé en février 1966 en vue de la préparation du plan directeur de la région métropolitaine. Ce bulletin intitulé Caractéristiques physiques comporte diverses planches qui donnent un bon aperçu des conditions physiques régionales.
} 
on en retrouve aussi plusieurs dans la «péninsule» Vaudreuil-Soulanges. En 1831, la région compte environ 252000 habitants dont un peu plus de 27000 vivent à la ville. À elles seules, les campagnes en accueillent 225000 , répartis pour près de la moitié sur la rive sud du fleuve où les effectifs humains dépassent les 119000 habitants. Quant aux densités rurales, elles sont de l'ordre de 18 habitants au $\mathrm{km}^{2}$, mais avec des variations dans l'espace, qui rendent compte des contextes démographiques très divers dans lesquels évolue alors l'agriculture ${ }^{5}$.

En effet, même si la population reste partout nombreuse, les densités rurales varient considérablement d'un secteur géographique à l'autre. Plus élevées dans l'île de Montréal (ville exclue) et l'île Jésus où elles gravitent autour de 32 à 37 habitants au $\mathrm{km}^{2}$ en moyenne (et ce, en dépit d'une population globalement peu élevée, malgré des maisonnées moyennes assez fortes), les densités rurales varient de 20 à 26 sur les rives du fleuve et dans la vallée du Richelieu où pourtant la population est nettement plus nombreuse, pour s'abaisser à 15 ou 16 dans les paroisses de l'intérieur et à 11 ou 12 dans l'arrière-pays seigneurial, le cas de la Petite Nation devant être considéré à part en raison de l'étendue de son territoire et d'un contexte pionnier où les défrichements, encore peu avancés, obligent à des concentrations temporaires dans l'espace (tableau 1). Calculées sur la base de l'espace réellement occupé, les densités y seraient plus élevées, de l'ordre d'une vingtaine d'habitants au $\mathrm{km}^{2}$ environ. Il n'y a donc pas nécessairement de correspondance ici entre le nombre total d'habitants retrouvés dans un secteur donné et leur densité brute dans l'espace. Il n'y a pas non plus de correspondance entre cette densité et les densités réelles des côtes. C'est que, loin d'être uniformément répandue dans le territoire, la population se distribue selon un gradient qui varie en fonction de l'ancienneté du peuplement et de la présence sur place d'un village qui en absorbe une part plus ou moins importante.

\section{Les structures d' habitat}

Même si le contexte premier de l'établissement rural reste la côte (appelée aussi «concession» ou «rang» dans les sources), les villages (entendre les bourgs) sont partout nombreux et en pleine croissance,

5 Jusqu'à tout récemment ce calcul (non plus d'ailleurs que celui des densités agricoles, comme on le verra plus loin) n'était pas possible, le dessin (c'est-à-dire les limites et la superficie) des territoires recensés n'étant pas connu, sauf pour quelques localités ayant fait l'objet d'études de cas spécifiques. C'est pourquoi il a fallu, avant d'entreprendre l'étude de l'agriculture dans la région de Montréal, refaire l'histoire territoriale des localités recensées et dépouiller systématiquement les listes nominatives de recensement pour localiser le plus précisément possible les populations dénombrées. Quant à la superficie des côtes et des localités recensées, elle a été obtenue par informatique, à partir des fonds de cartes réalisés dans le cadre de nos travaux sur les divisions administratives anciennes du territoire. Voir S. Courville, dir., J. Crochetière, P. Désaulniers et J. Noël, Paroisses et municipalités de la région de Montréal au XIXe siècle (18251861) (Québec, Presses de l'Université Laval, 1988), 350 p. 
tableau 1

LA POPULATON REgamale

\begin{tabular}{|c|c|c|c|c|c|c|c|c|c|c|c|c|c|}
\hline $\begin{array}{l}\text { Sous-roglone } \\
\text { locallites }\end{array}$ & \begin{tabular}{|} 
nor. de ch. de menages \\
des localites
\end{tabular} & $\begin{array}{c}\text { pop. totale } \\
\text { des mireges }\end{array}$ & menage & densites au $\mathrm{km} 2$ & $\begin{array}{c}\text { nor. de soviturse } \\
\text { termiers }\end{array}$ & \begin{tabular}{|c|} 
nor. do productours \\
agicoles
\end{tabular} & en $x$ do la localite & \%o rancoph. & \% propristaires & \begin{tabular}{|l|} 
pop. wotalo \\
des menegese
\end{tabular} & pop/manage & \begin{tabular}{|c|} 
surlace occupee \\
densite agricole \\
onnte au $\mathrm{km}^{2}$ de
\end{tabular} & \begin{tabular}{|l} 
surface culfive \\
densite agricole \\
netro au km2 do
\end{tabular} \\
\hline 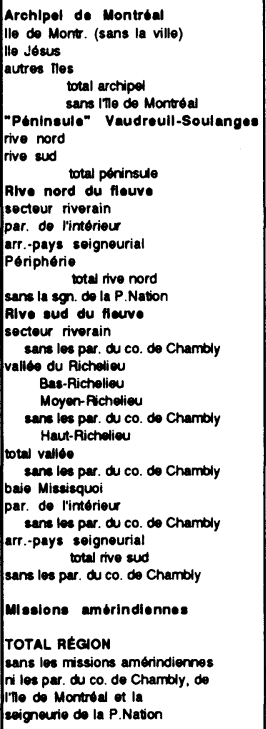 & $\begin{array}{r}2729 \\
1326 \\
235 \\
4290 \\
1561 \\
839 \\
8178 \\
2017 \\
4335 \\
5310 \\
2368 \\
152 \\
12166 \\
12014 \\
4966 \\
3661 \\
1465 \\
3614 \\
2520 \\
2109 \\
7188 \\
6094 \\
492 \\
6656 \\
6056 \\
1020 \\
20322 \\
17323 \\
351 \\
39146 \\
32915\end{array}$ & 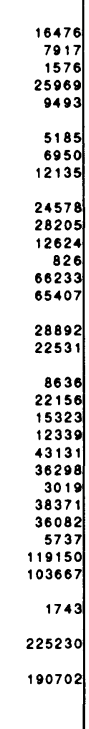 & $\begin{array}{l}6.04 \\
5.97 \\
6.71 \\
6.05 \\
6.08 \\
6.18 \\
5.90 \\
6.02 \\
5.67 \\
5.34 \\
55.33 \\
55.43 \\
5.44 \\
5.44 \\
6.82 \\
6.15 \\
5.89 \\
6.13 \\
65.08 \\
55.85 \\
55.00 \\
6.96 \\
5.76 \\
55.96 \\
5.62 \\
55.86 \\
5.98 \\
4.97 \\
5.75 \\
5.79\end{array}$ & $\begin{array}{l}36.45 \\
32.58 \\
24.25 \\
34.17 \\
30.82 \\
13.64 \\
19.86 \\
16.62 \\
21.58 \\
16.58 \\
11.00 \\
1.28 \\
14.28 \\
16.40 \\
26.48 \\
26.89 \\
24.08 \\
26.9 \\
23.54 \\
16.32 \\
21.98 \\
20.55 \\
14.73 \\
15.04 \\
14.48 \\
12.47 \\
19.00 \\
18.00 \\
13.41 \\
17.98 \\
17.68 \\
17.68\end{array}$ & $\begin{array}{r}591 \\
321 \\
23 \\
935 \\
344 \\
70 \\
98 \\
168 \\
348 \\
250 \\
115 \\
42 \\
755 \\
713 \\
688 \\
556 \\
28 \\
637 \\
537 \\
144 \\
809 \\
709 \\
72 \\
350 \\
331 \\
35 \\
1954 \\
1703 \\
0 \\
031 \\
3812 \\
2928\end{array}$ & $\begin{array}{r}2779 \\
1204 \\
2079 \\
1612 \\
4895 \\
295 \\
5121 \\
864 \\
13954 \\
295 \\
24948 \\
24653\end{array}$ & $\begin{array}{l}75.91 \\
82.18 \\
82.50 \\
76.43 \\
80.32 \\
50.96 \\
84.56 \\
84.71 \\
80.55 \\
84.05 \\
63.73 \\
74.90\end{array}$ & $\begin{array}{c}\text { n.9. } \\
97.45 \\
96.55 \\
97.28 \\
88.95 \\
85.68 \\
86.84 \\
88.62 \\
93.9 \\
89.92 \\
\text { n. } \\
91.19 \\
96.58 \\
93.44 \\
96.87 \\
57.32 \\
83.00 \\
0.09 \\
84.32 \\
92.94 \\
85.05 \\
9.83 \\
86.82 \\
87.74\end{array}$ & $\begin{array}{r}81.05 \\
87.93 \\
82.45 \\
85.53 \\
81.5 \\
82.97 \\
88.38 \\
94.2 \\
89.43 \\
0.0 . \\
90.49 \\
75 \\
75.59 \\
81.48 \\
88.9 \\
85.61 \\
77.50 \\
91.19 \\
80.33 \\
85.30 \\
78.93 \\
72.20 \\
82.94 \\
83.07\end{array}$ & 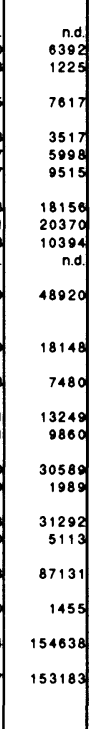 & $\begin{array}{l}6.53 \\
6.21 \\
6.37 \\
6.12 \\
6.25 \\
6.74 \\
6.11 \\
5.92 \\
6.24 \\
4.23 \\
6.20 \\
6.21\end{array}$ & $\begin{array}{c}n 0.8 \\
50.52 \\
26.10 \\
43.91 \\
26.08 \\
26.69 \\
26.46 \\
29.97 \\
21.24 \\
25.17 \\
n 0 . \\
24.74 \\
\\
30.91 \\
29.59 \\
26.62 \\
23.84 \\
26.28 \\
12.92 \\
16.97 \\
22.96 \\
21.94 \\
166.70 \\
23.82 \\
23.63\end{array}$ & $\begin{array}{c}56.85 \\
48.53 \\
56.18 \\
41.68 \\
53.09 \\
n .0 \\
48.54 \\
49.52 \\
78.32 \\
43.96 \\
68.63 \\
56.14 \\
26.86 \\
54.46 \\
72.60 \\
53.44 \\
426.22 \\
52.41 \\
51.98\end{array}$ \\
\hline
\end{tabular}

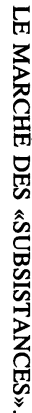


réunissant plus de 28000 habitants en 1831 (sans les missions amérindiennes), soit l'équivalent de la population de la ville de Montréal. Au total, on en compte plus d'une centaine (contre moins du quart en 1815), dont quatre atteignent ou dépassent le millier d'habitants: Berthier, sur la rive nord du fleuve, et Laprairie, Saint-Hyacinthe et Sorel, sur la rive sud. Plusieurs en comptent de 500 à 1000 , tels Saint-André d'Argenteuil, l'Assomption et Saint-Eustache, sur la rive nord, et Christieville, Boucherville, Varennes, Châteauguay et Saint-Jean sur la rive sud. Quant aux autres, ils sont moins populeux, mais comptent tout de même entre 100 et 500 habitants, seuls une douzaine d'entre eux en comprenant moins de 100 . Ensemble, ils regroupent de $15 \%$ à $16 \%$ de la population qui vit à la campagne, dans des proportions qui varient toutefois elles aussi dans l'espace: 14,93\% dans l'archipel montréalais, $11,87 \%$ dans la «péninsule» Vaudreuil-Soulanges, 16,16\% sur la rive nord du fleuve et $15,17 \%$ sur la rive sud.

Ces agglomérations se présentent sous des formes très diverses et ont des fonctions qui les font paraître tantôt comme des produits de la campagne, tantôt comme des produits de la relation ville-campagne, des relais, dans le territoire, de l'économie et des valeurs urbaines. En outre, selon leur localisation dans l'espace, elles ont un rôle éminemment variable, plus humble si le village vit à l'ombre de gros bourgs, plus important s'il est situé à un carrefour stratégique. Aussi la diversité est-elle de règle, certains bourgs n'étant que de petits centres de services pour les campagnes environnantes, d'autres, des centres actifs de commerce dont l'aire d'influence s'étend loin dans l'espace, d'autres encore, de simples hameaux construits autour d'un moulin ou d'importants lieux de production réunissant une part notable des industries de la campagne ${ }^{6}$.

Partout présents, les bourgs n'occupent pourtant qu'une partie du territoire de la localité, l'autre étant le domaine de la côte, c'est-à-dire de ces longues lignes d'habitat semi dispersé dont le naturaliste suédois Pehr Kalm disait qu'elle ressemblait à de longs villages continus dans l'espace $^{7}$. Cette image est encore vraie en 1830, plus même qu'en 1749, en raison de densités humaines accrues et d'une multiplication des habitations depuis le milieu du 18e siècle. Toutefois, elle est plus juste pour les côtes riveraines (les «Grandes-Côtes», les «Côtes-du-Bord-de-l'Eau» ou les «Premiers-Rangs») que pour les côtes intérieures où ces densités

6 Voir Serge Courville, «Croissance villageoise et industries rurales dans les seigneuries du Québec, 1815-1851», Normand Séguin et François Lebrun, dir., Sociétés villageoises et rapports villes-campagnes au Québec et dans la France de l'Ouest, XVIIe-XXe siècles. Actes du colloque franco-québécois de Québec, 1985 (Trois-Rivières, Centre de recherches en études québécoises, 1987), 205-219.

7 Sur l'oeuvre de Pehr Kalm, voir Jacques Rousseau et Guy Béthune avec le concours de Pierre Morisset, Voyage de Pehr Kalm au Canada. Traduction annotée du journal de route (Montréal, Pierre Tisseyre, 1977), $674 \mathrm{p}$. 
sont presque toujours moins élevées. En outre, rares sont les côtes situées à moins de quelques heures de marche d'un village. Un nouveau «rang» apparaît-il que très tôt un hameau se développe, lequel réduit la distance des écarts ${ }^{8}$. Aussi le village est-il partout présent, même sur les fronts pionniers où il accompagne le peuplement qu'il précède en certains cas.

\section{Les voies de communication}

Ce qui frappe aussi dans le paysage régional, c'est la quantité et la densité de voies de communication dont la campagne est dotée, qu'elles soient naturelles comme les cours d'eau ou fabriquées de main d'homme comme les routes. La principale voie de circulation reste le Saint-Laurent qui accueille une importante vie de relation. L'iconographie ancienne en témoigne, montrant quantité de radeaux, barges, bacs, chalands, chaloupes, voiliers, vapeurs qui remontent ou descendent le fleuve, ou qui vont d'une localité ou d'une rive à l'autre, et qui illustrent la variété d'échanges qui s'établissent alors dans le territoire. Suivent, dans l'ordre, la rivière Richelieu, dont le rôle dans l'économie du blé est connu, et la rivière des Outaouais qui donne accès aux chantiers du nord-ouest du Bas-Canada et de la région de Bytown (Ottawa). Les autres cours d'eau ont des fonctions plus locales. Peu d'entre eux sont réellement navigables, quelques-uns sont flottables, beaucoup cependant sont propices aux petites industries rurales qui viennent s'y installer nombreuses, partout où existe un seuil facile à endiguer et où les débits sont stables, ne souffrant pas trop des inondations printanières ni des étiages d'été. Â côté de tous ces «chemins qui marchent», il y a aussi ceux que l'État, le seigneur ou le paysan lui-même ont construits: chemins du roi, chemins de rangs, montées, simples sentiers, etc., qui ajoutent aux voies d'eau quantité d'autres moyens d'accéder au marché et dont la cartographie de l'époque nous donne un assez bon aperçu. Plusieurs ne sont que de mauvais chemins de terre, «billotés» par endroits, dont les habitants se plaignent régulièrement; d'autres, des chemins de sable et de gravier, dont le fond est meilleur mais dont l'entretien laisse tout autant à désirer, dépendant du bon vouloir de chacun. Tous n'ont pas les mêmes fonctions ni la même circulation, mais tous jouent un rôle indéniable dans la vie d'échange. Les revendications de l'époque en témoignent ${ }^{9}$, tout comme le dessin lui-même

\footnotetext{
8 Sur la notion d'écart et la définition des formes de groupement et de dispersion en milieu rural, voir Jean Tricart, L'habitat rural (Paris, Sorbonne, 3e édition, cours de l'Université de Strasbourg, Centre de documentation universitaire, s.d.), 175 p.

9 On en a un exemple dans cet extrait de lettre du docteur Jacques Labrie à Robert Neilson (16 janvier 1827): «Il est devenu presque impossible de transporter aucuns de nos produits au marché et que nous ne pouvons non plus rien avoir de la ville. Nos chemins sont garnis d'ici à la ville de voitures cassées, un de nos citoyens la semaine derniere a mis quatre heures avec un bon cheval et une voiture bien legere pour faire les deux lieues qu'il y a depuis St-Laurent à Montréal. Nos habitans ne font aucun argent, parce qu'ils ne peuvent aller au marché; tout le monde est en souffrance.» Cité par Fernand Ouellet dans Histoire économique et sociale du Québec, 17601850 , 2: 363-364. Toutefois, si cette lettre montre bien les difficultés posées par les chemins, surtout l'hiver ou après une pluie, elle montre aussi l'attitude des cultivateurs face aux échanges.
} 
du réseau routier qui montre, à côté des chemins parallèles au fleuve et à ses principaux affluents, quantité d'autres tracés perpendiculaires dont le rôle est autant d'assurer la liaison entre les rangs et entre les rives et l'intérieur des terres. On le voit très nettement dans l'habitat de certains bourgs riverains où entrepôts, greniers, hangars de toutes sortes jouxtent des quais ou des débarcadères achalandés. On le voit aussi dans la quantité de ponts qui relient ces routes entre elles, ponts de pierre, ponts de bois, ponts payants, ponts libres, que remplacent, sur les cours d'eau importants, des traverses l'été et des ponts de glace l'hiver.

Bref, quel que soit l'angle sous lequel on l'observe, la région semble assez bien servie en voies de communication. Et si la construction et l'entretien des routes restent à l'époque un problème permanent dont la législature est régulièrement saisie ${ }^{10}$, il n'en demeure pas moins qu'elles sont complémentaires du rôle que jouent le fleuve et ses principaux affluents dans la vie de relation, contribuant à définir les circuits locaux d'échange et à désenclaver les localités de l'intérieur. Surtout, elles offrent à l'agriculture un moyen d'accéder au marché, car si la ville reste dans bien des cas une réalité éloignée, du moins sur le plan géographique, le village, lui, est beaucoup plus accessible, devenant ainsi l'un des principaux paliers où se nouent ces échanges. Or, c'est par la route généralement qu'on y accède même quand la voie d'eau est toute proche, ce qui peut expliquer sans doute bien des récriminations, puisque, lorsqu'elle fait défaut, c'est l'accès au marché qui est compromis (figure 1).

\section{LES INDUSTRIES RURALES}

$\mathrm{Au}$ Bas-Canada, le démarrage industriel a pris trois grandes directions reliées, l'une, à l'exploitation forestière et au commerce du bois (scieries, chantiers maritimes), l'autre, à la fabrication manufacturière des villes, et la troisième, à la poussée d'ateliers et de fabriques enregistrée à la campagne ${ }^{11}$. C'est cette dernière direction qui nous intéresse

10 En 1828-1829, notamment, au moment où la législature forme un comité chargé d'enquêter sur l'état des chemins et sur les améliorations à apporter au réseau routier. Voir les Journaux de l'Assemblée législative du Bas-Canada (JALBC) de 1828 à 1829, app. U, et la carte de William Sax, Figurative Plan Exhibiting a Bird's Eye View of the River St.Lawrence and Its Principal Tributary Streams From Kingston in Upper Canada to Blanc Sablon on the Labrador Coast [...]: Principally Intended to Elucidate Evidence Given Before a Committee of the Honorable House of Assembly for Internal Communications, in February 1829. Archives nationales du Canada, collection nationale de cartes et plans, 300/1829, 19110.

11 Pour une présentation de ces orientations, voir Stanley-Bréhaut Ryerson, Le Capitalisme et la Confédération (Montréal, Éditions Partis Pris, 1978), 31-33 (l'édition originale date de 1968). Voir aussi Fernand Ouellet, Histoire économique et sociale du Québec [...] (1971), 2 volumes. On en trouvera également des éléments dans John McCallum, Unequal Beginnings, Agriculture and Economic Development in Quebec and Ontario Until 1870 (Toronto, Buffalo, London, University of Toronto Press, 1977), 148 p. Quant au rôle des hommes d'affaires dans le développement de l'industrie urbaine, à Montréal notamment, voir Gerald J. J. Tulchinsky, The River Barons. Montreal Businessmen and the Growth of Industry and Transportation, 1837-53 (Toronto et Buffalo, University of Toronto Press, 1977), $310 \mathrm{p}$. 
L'HABITAT ET LEg ROUTEB (1831)
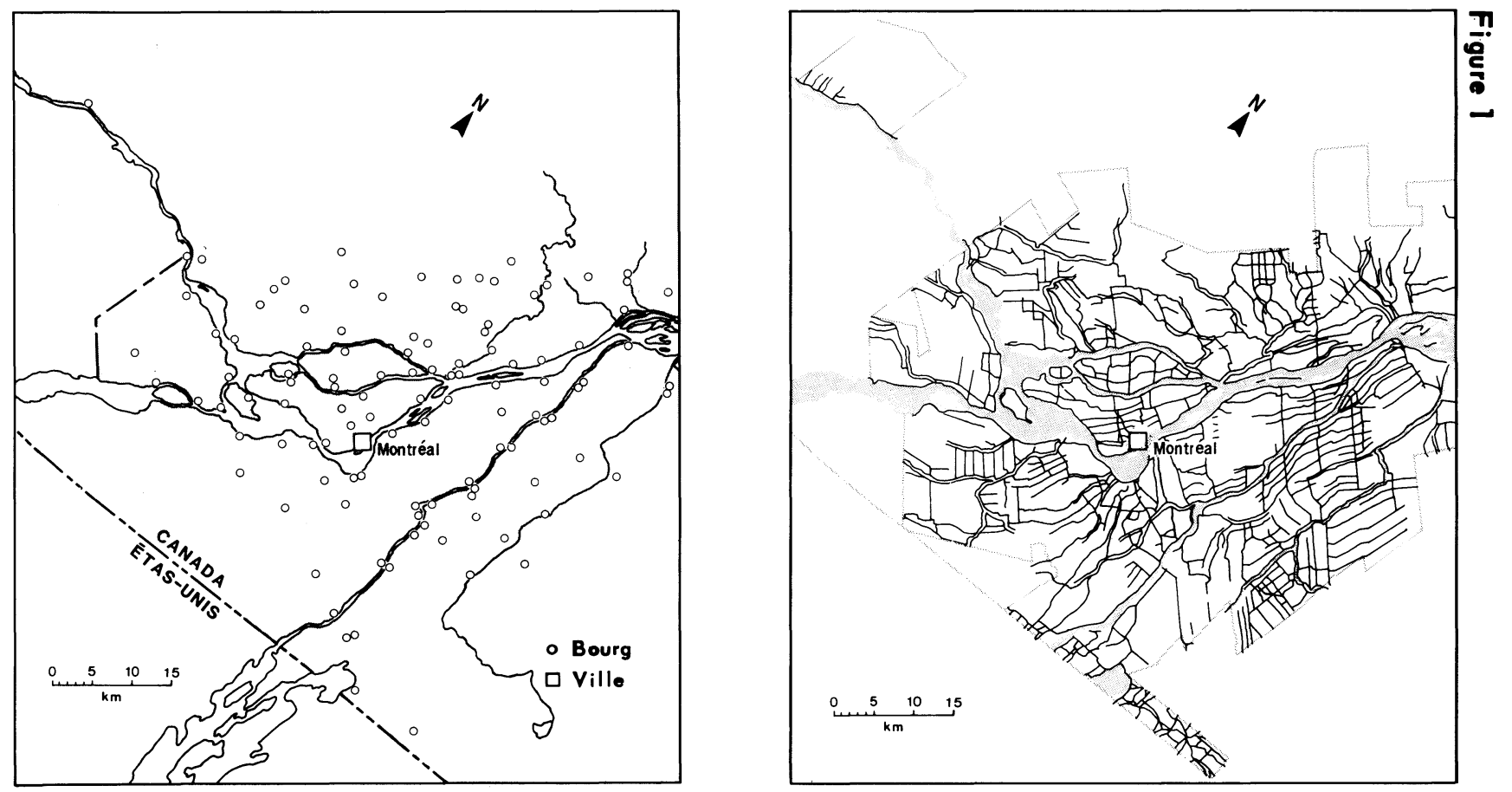

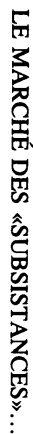

Source: Recensement du Canada, 1831 
ici, conjuguée en partie avec la première pour tenir compte du rôle qu'a joué l'exploitation forestière dans la prolifération des petits moulins à scie.

Par industries rurales, nous entendons des productions qui trouvent à la campagne le lieu de leur réalisation. Ces industries sont à distinguer de la simple production artisanale: celles auxquelles nous nous référons mobilisent des investissements en capitaux, recrutent sur une base saisonnière ou annuelle des travailleurs salariés, issus ou non du monde agricole, et dépendent pour leurs opérations de réseaux parfois denses d'approvisionnement dans l'espace. Ce sont les moulins (à farine $^{12}$, à scie, à fouler, à carder, à huile, à papier, à bardeaux, etc.), les brasseries, distilleries, tanneries, fabriques de potasse ou de perlasse, ateliers et manufactures de toutes sortes qui emploient parfois une importante main-d'oeuvre ${ }^{13}$ dont ne font pas toujours état les sources documentaires de la période et qui renvoient à des organisations économiques spécifiques, distinctes de l'artisanat domestique, mais qui peuvent aussi s'y alimenter à l'amont ${ }^{14}$. Certaines, nées de l'initiative locale, évoluent dans des rapports étroits avec la paysannerie; ce sont les plus nombreuses. Elles sont de taille modeste et intimement reliées à la satisfaction des besoins locaux ou sous-régionaux. D'autres, nées d'initiatives capitalistes, sont de taille plus imposante et s'appuient pour leur croissance aussi bien sur la campagne que sur la ville. Elles sont beaucoup moins répandues et concentrées surtout autour des villes; elles ne se multiplieront vraiment que dans la seconde moitié du siècle ${ }^{15}$.

L'origine de ces industries remonte loin dans le temps. On en retrouve la trace dès le Régime français, aux endroits mêmes où les

12 Parce que son exploitation s'inscrit dans le cadre de monopoles reliés aux titres des seigneurs, le moulin à farine ne constitue pas comme tel une «industrie rurale». Toutefois, comme son bâtiment abrite souvent des machines qui le sont (moulins à carder par exemple), ou des scies utilisées pour le débitage des billots, il est à ranger parmi celles-ci, d'autant plus qu'après les années 1815-1820 nombreux sont les marchands ou hommes d'affaires urbains qui achètent des seigneuries pour y exploiter des moulins.

${ }_{13}$ Au bourg Saint-Denis, par exemple, dans la vallée du Richelieu, la fabrique de chapeaux (de castor) fondée par Charles Saint-Germain en 1825 emploie une trentaine d'ouvriers, la distillerie appartenant aux docteurs Nelson et Kimber et à Louis Deschambault emploie une douzaine d'hommes, la fabrique de voitures de François Gadbois, une dizaine, sans oublier ceux qu'occupent la carderie, les poteries et les autres petites entreprises enregistrées dans les sources. Voir Stanley-Bréhaut Ryerson, Le Capitalisme et la Confédération..., 32. La même situation prévaut dans d'autres villages, tel celui de Saint-Eustache, où Bouchette rapporte la présence d'une fabrique de potasse, une poterie, deux tanneries, une manufacture de cigares et de tabac «in great repute», et une fabrique de chapeaux et une de chaises «all enjoying considerable reputation», mais dont il ne précise pas le nombre d'employés. Joseph Bouchette, A Topographical Dictionary of Lower Canada (London, Rees, Orme, Brown, Green and Longman, 1832), n.p., «Mille Isles».

14 Voir S. Courville, «Croissance villageoise et industries rurales [...]», et René Hardy, Pierre Lanthier et Normand Séguin, «Les industries rurales et l'extension du réseau villageois dans la Mauricie pré-industrielle: l'exemple du comté de Champlain durant la seconde moitié du 19e siècle», François Lebrun et Normand Séguin, Sociétés villageoises et rapports villes-campagnes [...], 205-219 et 239-253.

15 John McCallum, Unequal Beginnings [...], 83ss. 
seigneurs avaient entrepris d'établir leur seigneurie (leur domaine souvent) ou dans leur voisinage. Ce qui change toutefois au 19e siècle, c'est l'ampleur de leur croissance. En 1815, par exemple, Bouchette en signale déjà plusieurs dans le territoire seigneurial, sous forme de moulins à farine, de moulins à grain et de moulins à scie, auxquels viennent s'ajouter quelques moulins à fouler et à carder, et quelques fabriques de potasse. En 1831, elles sont plus nombreuses encore, élargies cette fois à toute une gamme d'activités dont les recensements ne donnent qu'un aperçu. Bouchette en a laissé d'ailleurs des images saisissantes, telle cette description des moulins Harrower dans la seigneurie de Saint-Jean-Port-Joli:

At the mouth of the river Trois Saumons the valuable mills and distillery belonging to Mr. Harrower are very eligibly placed: the latter is an establishment of considerable magnitude, with every convenience of carrying on an extensive business; at high water decked vessels of twenty tons may come up the premises. Over the river is a good bridge. [...] From the St Lawrence the view of the mills and surrounding objects, heightened by the pleasing natural scenery of the environs is very agreable. ${ }^{16}$

En 1831, on dénombre quelque 2000 moulins et fabriques dans l'ensemble du Bas-Canada, dont plus des deux tiers sont localisés dans le territoire seigneurial et plus de la moitié dans la seule région de Montréal. De ce nombre, environ 640 sont situés dans le territoire à l'étude, auxquels il faut ajouter la quarantaine de tanneries, les douzaines de poteries et la demi-douzaine de fabriques (de chapeaux, de chaises, de tabac, etc.) signalées par Bouchette dans son dictionnaire topographique de 1832 , mais que le recensement ne relève qu'en partie sous la mention «fabriques de toutes autres espèces» ${ }^{17}$ (tableau 2 ). Dans les cantons, où le caractère récent de la colonisation associé à l'importance de l'exploitation forestière et de l'élevage stimulent également cette poussée industrielle, on en compte déjà près de 490 dont le quart environ sont des moulins à scie et plus de $45 \%$ des fabriques de potasse et de perlasse, auxquelles s'ajoutent une trentaine de moulins à fouler et à carder et autant de distilleries, en raison de la présence toute proche des chantiers. Omniprésente, l'industrie rurale imprime partout sa

\footnotetext{
16 Joseph Bouchette, A Topographical Dictionary [...], «St-Jean-Port-Joli».

17 Sauf pour les localités des comtés de Montréal et de Chambly, dont les listes nominatives ont été perdues ou égarées, tous nos relevés ont été effectués sur une base nominale, sans tenir compte de l'information statistique contenue dans les tableaux récapitulatifs des commissaires, qui rapportent parfois plus de moulins ou de fabriques que n'en comportent les listes. Nos données sont donc un minimum. Elles comprennent toutefois la quarantaine de fabriques de fer signalées dans le comté de Saint-Hyacinthe, qui ne sont probablement que de petites forges rurales. Mais comme la fabrication de matériel de transport et de pièces de moulins semble importante à l'époque, il se peut que quelques-unes d'entre elles soient des ateliers plus élaborés, pouvant être considérés comme des industries rurales.
} 
TABLEAU 2

LES INOUSTRIES RURALES

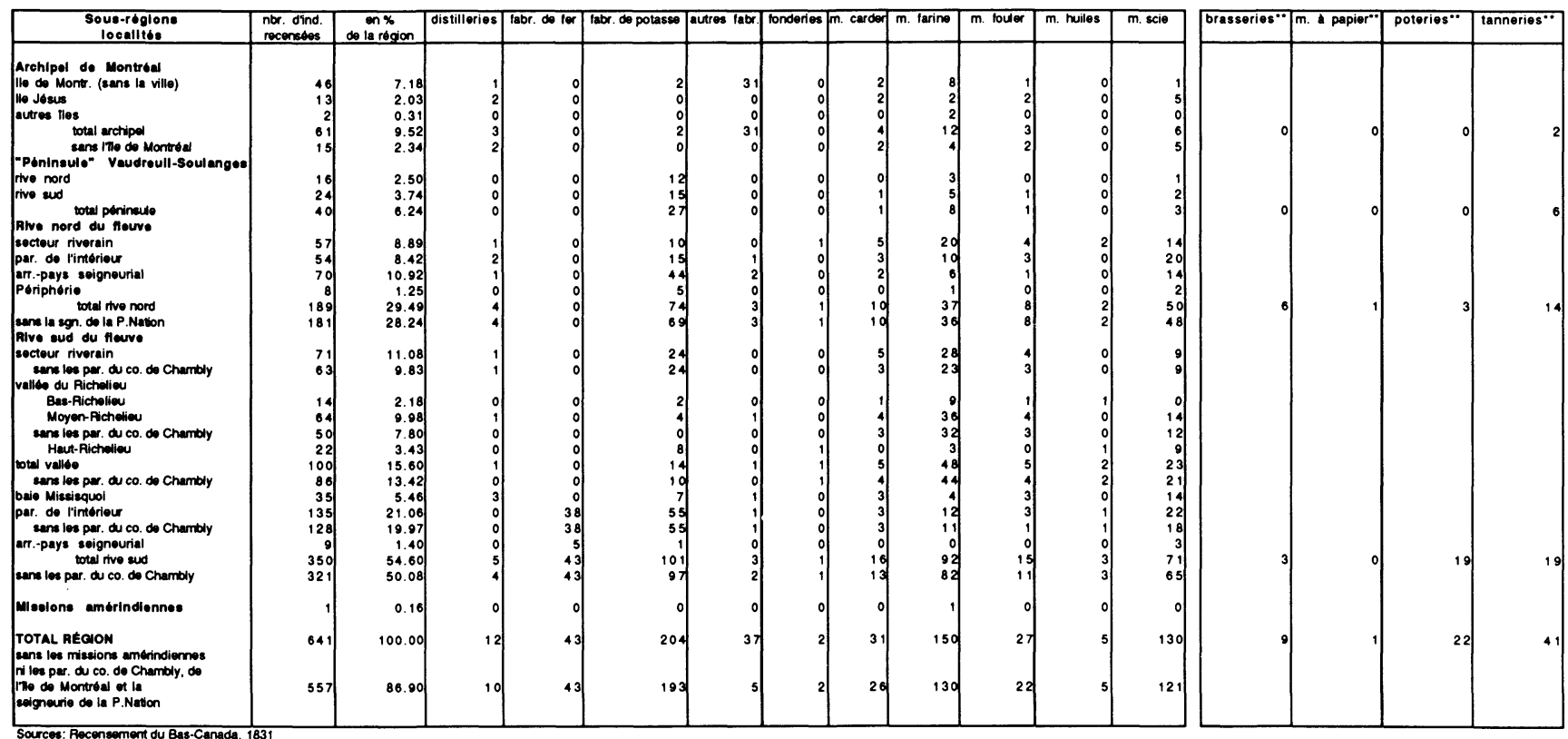

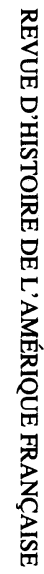

. Solon Joseph Bouchette, Dictiomaire Topographique du Bas-Canada (...), 1831.

N.B. Contrairement au tableau, la figure 2 níndique que les incustries mentom des dans les listes nominatives. 
marque, tant au village ${ }^{18}$ que dans les côtes, en un semis qu'illustre la figure 2 . La région à cartographier étant vaste et les industries nombreuses, il n'a pas été possible de les représenter toutes ${ }^{19}$, ni d'en indiquer les sites précis d'implantation au sein des entités territoriales recensées (bourgs, côtes, dont les moulins ou fabriques ont dû parfois être représentés à proximité). Toutefois, même si l'esquisse ici reste sommaire, elle montre bien comment se distribue alors l'industrie, la plupart des entreprises ayant tendance à se regrouper dans certaines aires bien localisées, dominant parfois tout un bassin-versant. On en a un exemple dans le comté de l'Assomption sur la rive nord de Montréal, à propos duquel, après avoir signalé la douzaine de moulins que compte le cours de la rivière l'Achigan, un affluent de la rivière l'Assomption, Jean-Baptiste Meilleur ajoute ce qui suit:

La Rivière de l'Assomption reçoit encore les eaux de la Rivière du Petit St. Esprit, sur laquelle sont construits un moulin à carder et une distillerie, à la distance d'environ trois milles du village de l'Assomption [...]. L'on (y) voit encore [...] plusieurs établissements dignes de remarques, et spécialement les beaux Moulins et le Village d'Industrie [...]. Sur la rivière du Lac Ouaro se trouve le beau moulin à farine des M.M. du Séminaire de St.Sulpice de Montréal, un moulin à carder, un moulin à faire des étoffes en laine et plusieurs moulins à scier le bois de construction. [Quant à la rivière Rouge voisine, elle accueille] le moulin florissant du Capt. P. Dugas et celui d'un dénommé Pratt dans le Township de Rawdon. ${ }^{20}$

En fait, cette situation est celle de tous les cours d'eau qui présentent un potentiel hydraulique intéressant, les plus recherchés demeurant les petites rivières au débit faible mais stable, plus faciles à endiguer et présentant moins de risques d'inondation au printemps ${ }^{21}$. Elle est d'autant plus répandue que, toutes proportions gardées, les îles de l'archipel de Montréal comptent peu de cours d'eau de ce genre, obligeant à une technologie différente (vg. le moulin à vent dont on retrouve aussi la

18 Le village au Bas-Canada est un important lieu de localisation d'industries rurales, regroupant parfois jusqu'au quart ou au tiers des industries de la paroisse. Si on élargissait l'aire d'observation à un demi ou à un kilomètre du bourg, le phénomène serait plus évident encore. Pour une démarche en ce sens, appliquée à la lecture des aires d'expression de la socio-économie villageoise, voir René Hardy, Pierre Lanthier et Normand Séguin, «Les industries rurales et l'extension du réseau villageois dans la Mauricie pré-industrielle», François Lebrun et Normand Séguin, Sociétés villageoises et rapports villes-campagnes [...], 239-253.

19 Certaines, dont l'existence nous était pourtant connue, n'ont pu être localisées, faute d'information sur leur lieu précis d'implantation; le tableau 2 toutefois en tient compte.

${ }_{20}$ Jean-Baptiste Meilleur, Extrait du recensement du comté de l'Assomption de l'année 1831 [...] (Montréal, Imprimerie de la Minerve), 10-11.

C'est le cas, entre autres, des rivières du Chêne et Chicot dans la seigneurie des MilleIsles, le long desquelles on ne compte pas moins d'une douzaine de moulins et de fabriques en 1831. C'est le cas aussi de la rivière Châteauguay et ses affluents, où le nombre de moulins et de fabriques dépasse la vingtaine. Au total, on compte ainsi une quinzaine de rivières dont les bassinsversants sont «industrialisés» dans la région de Montréal, incluant les rivières Richelieu et Yamaska. 


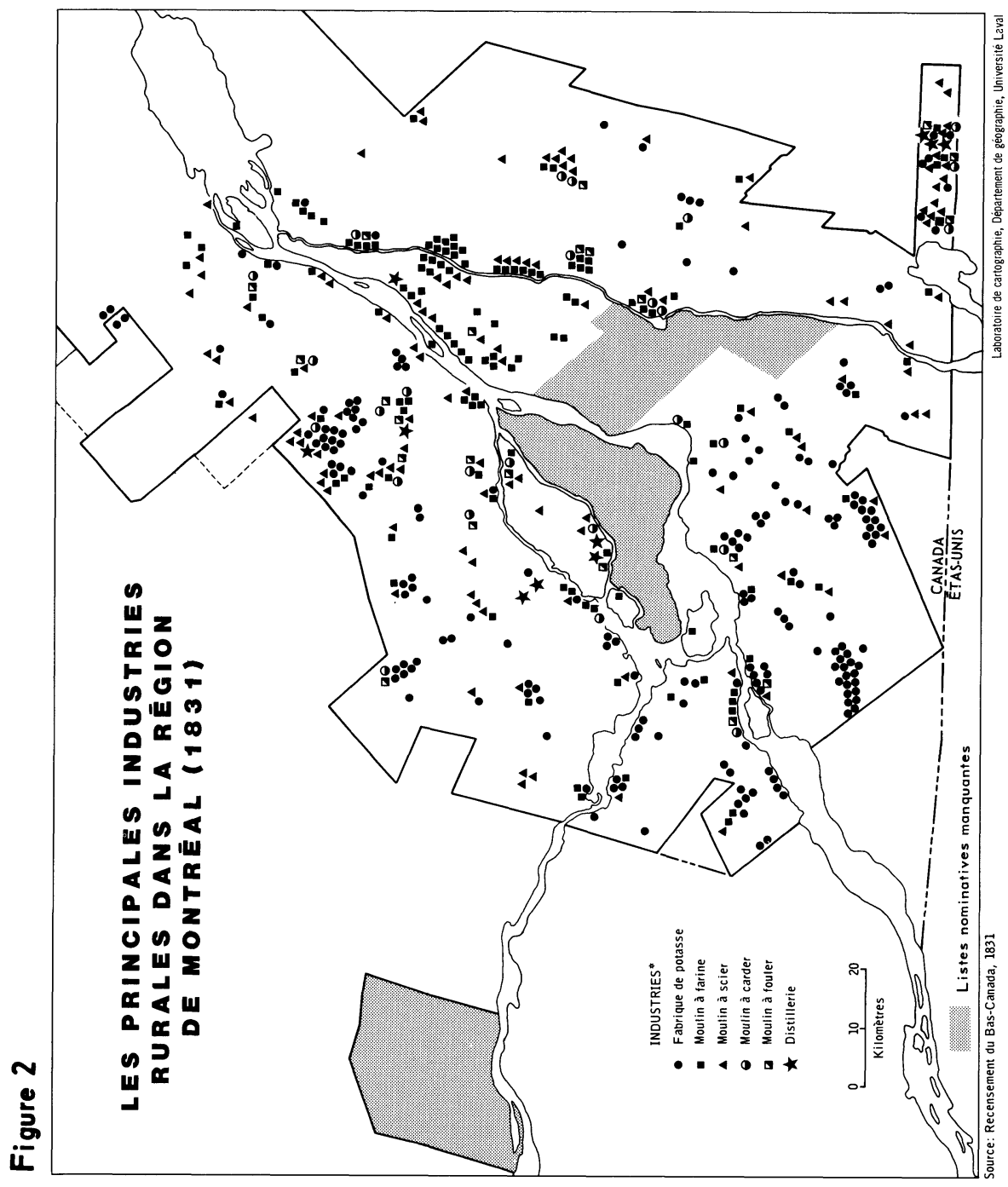

trace dans certains autres secteurs, le comté de Richelieu, par exemple), ou à des aménagements plus coûteux dont bien peu de petits entrepreneurs sont capables ${ }^{22}$. Aussi l'industrie gagne-t-elle la terre ferme, à la

22 C'est le cas, par exemple, au Sault-au-Récollet, où l'accès au moulin de l'île de la Visitation sur la rivière des Mille-Isles a nécessité des travaux importants dont on retrouve la trace sur tous les plans du Séminaire de Montréal relatifs à ce secteur. Mais ce n'est rien à côté des 
recherche de sites plus favorables, profitant de la vocation de certaines localités pour s'y établir et contribuant ainsi, par leur présence, à la renforcer.

Les moulins à farine, par exemple, sont partout nombreux mais plus sur la rive sud du fleuve que sur la rive nord où les terroirs sont moins riches. Les moulins à scie le sont tout autant, sinon plus, florissant partout où existe une forêt à exploiter ou un commerce à nourrir, que ce soit sur les fronts pionniers de l'intérieur ou de l'arrière-pays seigneurial, ou sur les rives du fleuve. Viennent ensuite les fabriques de potasse et de perlasse, dont la répartition suit de près les défrichements, puis les tanneries et les moulins à fouler et à carder, plus fréquents sur la rive nord. Quant aux brasseries (non enregistrées au recensement) et aux distilleries, elles sont beaucoup moins nombreuses. On les retrouve surtout sur la rive nord du fleuve et dans l'archipel montréalais. Mais on en trouve aussi quelques-unes sur la rive sud, à Contrecoeur, à Saint-Jean, et dans la seigneurie de Saint-Armand sur la baie Missisquoi.

Si l'industrie progresse tant, c'est que l'économie elle-même évolue, stimulée par les besoins nouveaux qu'entraînent la croissance démographique, l'extension de la demande intérieure et la meilleure insertion de la campagne dans les circuits d'échanges commandés par la ville. Aussi le paysage se transforme-t-il rapidement. Et si certains terroirs intérieurs paraissent plus démunis que d'autres en industries rurales, ces dernières ne sont jamais très loin, réparties ailleurs dans l'espace, au village notamment ou, si celui-ci est privé de cours d'eau pouvant alimenter les machines, dans les côtes ou les bourgs voisins, offrant à l'agriculture locale un débouché qui n'est pas sans intérêt puisque facilement accessible et complémentaire de ceux qu'introduisent la ville et le marché extérieur. Sans doute ce marché reste-t-il d'ampleur limitée, du moins sur le plan unitaire. Mais cumulé pour l'ensemble des industries de la campagne, celui-ci peut devenir intéressant, suffisamment pour susciter l'apparition de nouvelles combinaisons agricoles dont la logique n'apparaît qu'une fois prise en compte la présence d'industries à proximité. Pour saisir cette logique, il faut d'abord connaître les traits de l'agriculture régionale, puis la mettre en rapport avec le type d'industries rencontrées et leur localisation dans l'espace, non seulement à l'échelle de la région ou de la localité, mais à celle aussi de la côte. Car ce qui vaut pour un ordre de grandeur donné ne vaut pas nécessairement pour l'autre. Encore faut-il que les mêmes tendances se dessinent, quel que soit le territoire observée.

efforts qu'il a fallu consacrer pour l'aménagement du canal de Lachine. À ce sujet, voir Gerald J. J. Tulchinsky, The River Barons [...]; voir aussi John Willis, The Process of Hydraulic Industrialization on the Lachine Canal 1840-1880: Origins, Rise and Fall, rapport interne d'Environnement Canada, Parcs Canada, 2 volumes. 


\section{L'AGRICULTURE: SES GRANDS TRAITS}

L'une des plus sérieuses difficultés que pose l'étude de l'agriculture au Bas-Canada en est une de sources. Pourtant celles-ci ne manquent pas et si, comparé à certains pays européens, le Canada paraît avantagé, il faut reconnaître aussi que sa situation n'est pas sans problème $^{23}$. Par exemple, il n'est pas toujours possible d'exploiter à l'échelle régionale les documents utilisés pour les monographies locales (registres d'état civil, actes notariés, etc.), la masse de documents à traiter devenant ici beaucoup trop volumineuse. Par ailleurs, le pourrait-on que l'on se retrouverait vite aux prises avec les mêmes difficultés d'exploitation que rencontrent les chercheurs oeuvrant localement (séries discontinues dans le temps, couverture territoriale partielle, problèmes de représentativité, etc.). Aussi est-ce encore aux recensements qu'il faut avoir recours pour tenter de qualifier cette agriculture, d'autant plus qu'ils sont les seuls avec la carte et certaines oeuvres topographiques anciennes à autoriser une vue d'ensemble de cette activité dans l'espace et ce, pour un même moment et les mêmes variables ${ }^{24}$. Mais comme leurs limites sont nombreuses, on a eu tendance à les considérer comme des sources de second ordre, du moins avant 1851. En fait, on peut en tirer beaucoup d'informations valables, à condition de les aborder avec des stratégies appropriées d'exploitation. Car s'ils n'offrent pas toujours les renseignements voulus pour juger de l'état de l'agriculture à l'époque, ils en offrent suffisamment pour que l'on puisse en saisir les grands traits et même certaines particularités que confirment d'autres sources ${ }^{25}$. C'est le cas du recensement de 1831, dont les données ne sont pas toujours sans faille, mais qui, convenablement exploité, permet d'intéressantes observations.

23 À ce sujet, voir Louise Dechêne, «Observations sur l'agriculture du Bas-Canada au début du XIXe sièclę», Joseph Goy et Jean-Pierre Wallot, Évolution et éclatement du monde rural (Paris et Montréal, Éditions de l'École des Hautes Études en sciences sociales et Presses de l'Université de Montréal), 289-202.

24 Les travaux, ici, reposent sur trois sources principales: la première et la plus importante reste les listes nominatives du recensement de 1831 , complété de sa copie abrégée parue dans les $J A L B C$ de 1832, app. Oo. Dépouillées sur une base nominale, les données ont été agrégées par côtes, villages et localités, et saisies sur support informatique; la deuxième, utilisée surtout comme source d'atmosphère, est le dictionnaire topographique de Bouchette (1832), qui fournit pour chaque comté et chaque seigneurie quantité d'informations qui complètent avantageusement celles du recensement. La troisième est la cartographie de l'époque, plans de paroisses surtout et carte de Bouchette de 1831, dont le principal intérêt est d'offrir des renseignements sur les aménagements et la toponymie des localités. Ces outils ne sont pas toujours sans faille: le recensement de 1831, par exemple, fourmille d'erreurs d'addition, qu'alourdissent divers systèmes d'enregistrement des données dont plusieurs sont incomplètes ou mal insérées dans les listes. Le dictionnaire de Bouchette est une réédition revue mais non entièrement corrigée de sa Description topographique du Bas-Canada parue en 1815. Quant à sa carte de 1831 et aux plans de paroisses, ils omettent parfois bien des détails, les plus complets et les plus sûrs à cet égard demeurant les documents réalisés par des arpenteurs.

${ }_{25}$ Au recensement de 1831, par exemple, seules certaines grandes cultures sont enregistrées, sans les cultures fourragères, les pâturages, ni les cultures fruitières et maraîchères, dont on sent pourtant l'importance dans certaines localités à travers les mentions de Bouchette ou la taille des cheptels. 
Au début des années 1830, l'agriculture au Bas-Canada reste une activité largement répandue dans l'espace, qui occupe la majeure partie des ruraux, soit directement, soit indirectement. En outre, elle demeure une activité essentiellement familiale, dont les fonctions premières sont de satisfaire les besoins domestiques. Toutefois, comparée à ce qu'elle était encore à la fin du $18 \mathrm{e}$ siècle, bien des choses ont changé et si elle définit toujours un «genre de vie», elle n'est pas pour autant insensible aux transformations du contexte. En outre, si ses productions paraissent plus diversifiées qu'auparavant, lorsque le blé occupait plus des trois quarts de la récolte, elle a aussi ses «spécialités» qui la singularisent dans l'espace. Enfin, même si elle occupe encore une importante part des chefs de ménage, les variations locales sont nombreuses, montrant, encore là, qu'il n'y a pas nécessairement de correspondance entre les secteurs de fortes densités agricoles et ceux de fort peuplement ou de fortes densités rurales (figure 3 , tableaux 3 et 4 ).

\section{La population agricole}

Définir l'agriculture, c'est d'abord distinguer, au sein de la population rurale, la population agricole de la population non agricole, c'està-dire la population qui vit de cette activité de celle qui, tout en habitant la campagne, ne la pratique pas ou très peu, sinon pour agrémenter l'ordinaire ou pour nourrir les quelques animaux dont presque chaque ménage est pourvu. Cette distinction n'est possible dans les recensements canadiens qu'à partir de la seconde moitié du $19 \mathrm{e}$ siècle ${ }^{26}$. Or, même en 1830, on observe des écarts parfois importants entre les deux. Dans son dictionnaire topographique de 1832, par exemple, Bouchette rapporte plusieurs cas de seigneuries où une partie seulement des chefs de ménage vivent de l'agriculture ${ }^{27}$. Même l'État s'intéresse au problème, en s'enquérant du «nombre de familles qui gagnent leur subsistance par les travaux de l'Agriculture». Toutefois, comme la qualité d'enregistrement de cette information dans le recensement est très inégale d'une localité à l'autre ${ }^{28}$, la difficulté reste entière, obligeant à revoir la question sur d'autres bases. C'est ce que nous avons fait, en dépouillant cas par cas les déclarations individuelles des chefs de ménage pour établir cette distinction.

\footnotetext{
26 Et encore, puisque ce n'est qu'en 1921 que la ferme est définie dans les statistiques canadiennes. De 1851 à cette date, il faut s'en remettre aux recensements agraires qui incluent plusieurs «occupants de terre» sans récolte ou presque.

27 À Saint-Roch-des-Aulnaies, par exemple, seulement 186 familles sur 390 «are supposed to live entirely on the produce of their farms». Joseph Bouchette, A Topographical Dictionary $[\ldots]$, «St Roch des Aulnais».

${ }_{28}$ C'est ainsi, par exemple, que plusieurs chefs de ménage, qui se déclarent pourtant «cultivateurs» dans les listes et qui enregistrent des récoltes, ne sont pas toujours comptabilisés comme vivant de l'agriculture, alors que d'autres, ne se déclarant pas cultivateurs et ne déclarant que de faibles récoltes, le sont.
} 
Figure 3 a

\section{L'AGRICULTURE REGIONALE (1831)}

LES DENSITES HUMAINES
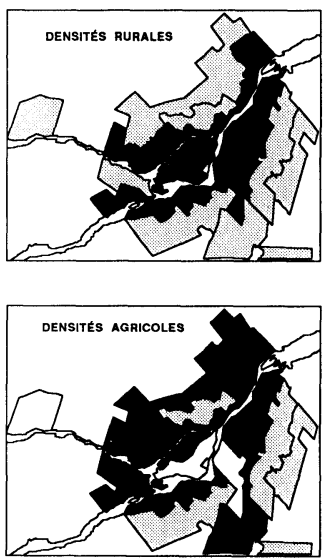

LA PROPRIETTE AGRICOLE
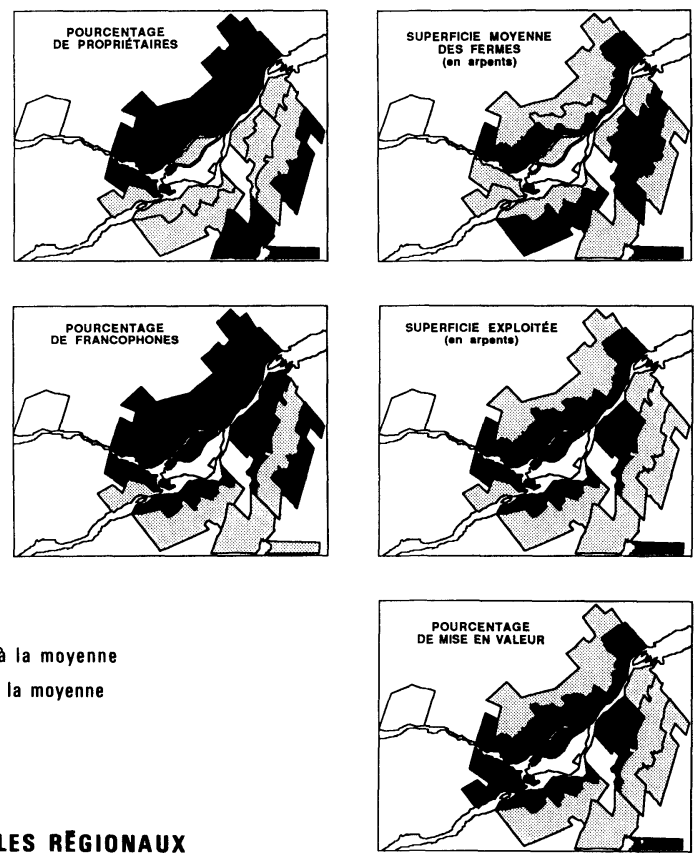

LES SOUS-ENSEMBLES REGIONAUX

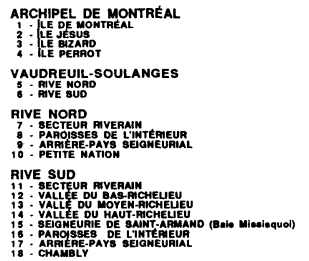

Source: Recensement du Bas-Canada, 1831

Le problème est beaucoup plus complexe qu'il ne paraît à première vue. D'une part, parce qu'à trop éliminer on risque de perdre de vue le nombre de ceux qui ne pratiquent qu'une petite agriculture difficile à distinguer souvent de la culture d'un jardin. Ensuite, parce qu'à tout prendre on risque d'attribuer à la masse des exploitants des traits qu'ils n'ont pas. On en a un exemple dans l'élevage, largement répandu à 
Figure 3b

\section{L'AGRICULTURE REGIONALE (1831)}

LES GRANDES CULTURES

(minots/exploitant)
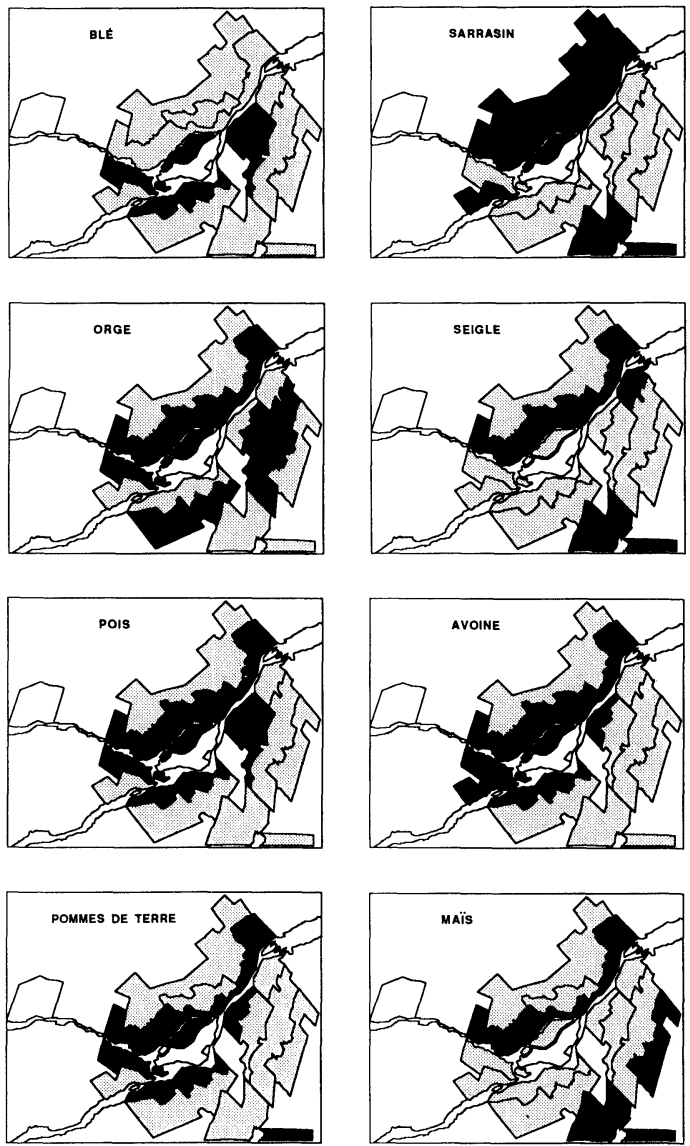

Supérieur à la moyenne

Inférieur à la moyenne

Source: Recensement du Bas-Canada, 1831

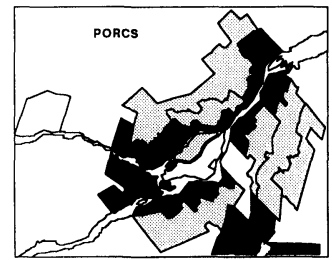

L'ELLVAGE

(moyenne/exploitant)
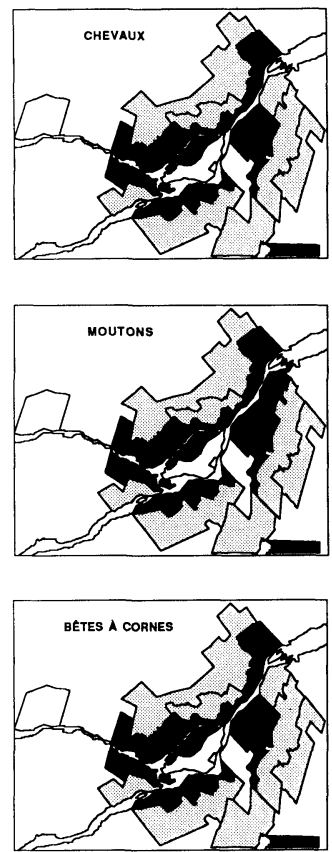

l'époque, mais qu'on ne peut toujours comptabiliser comme activité agricole, étant donné que la plupart des ménages possèdent des animaux même quand ils ne pratiquent pas d'agriculture. En général, les écarts sont assez nets entre les déclarations pour que l'on puisse distinguer ce 
TABleau 3

L'AGRICULTURE REGIONALE

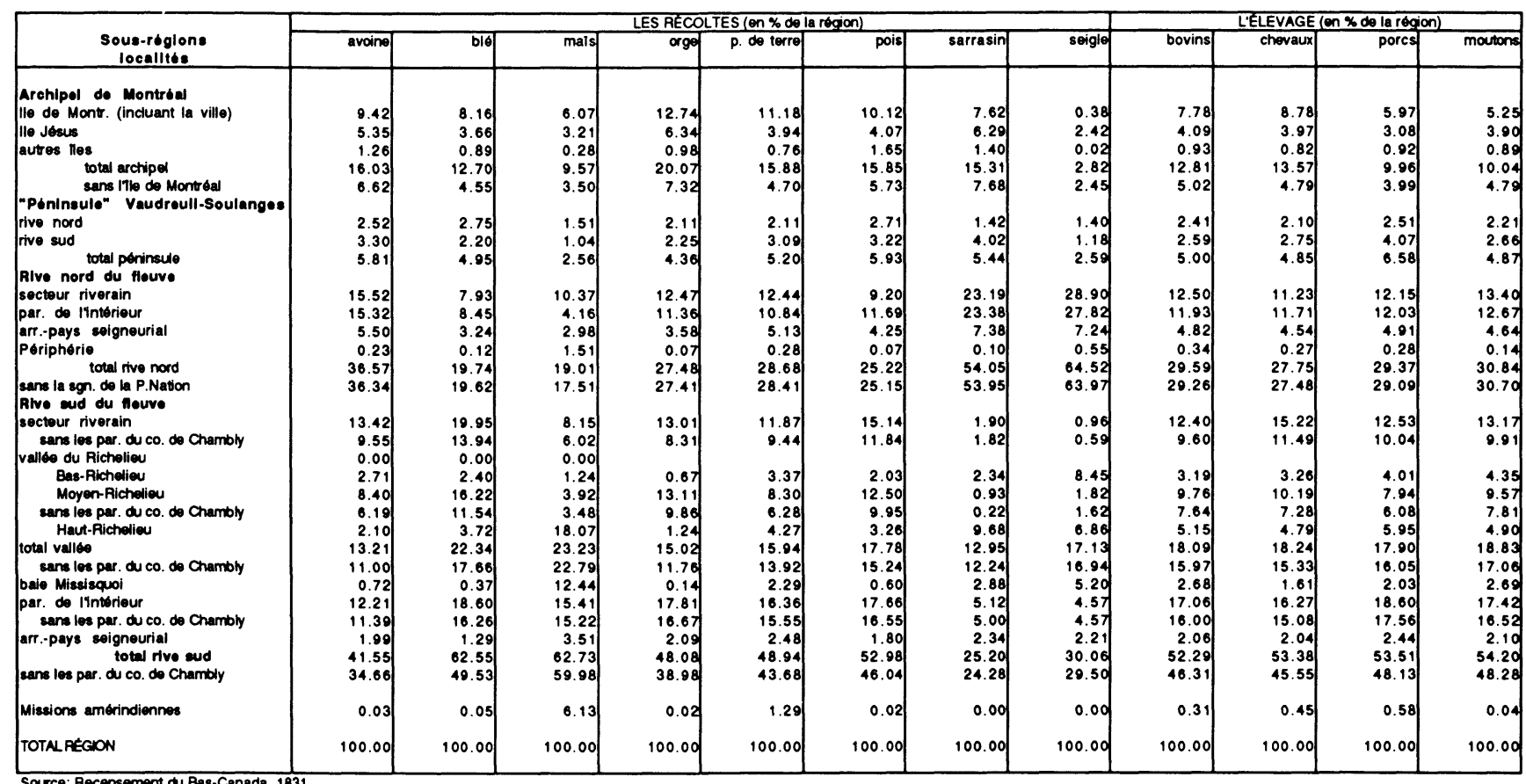

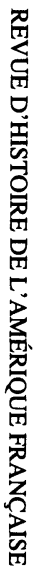


TABLEAU 4

TNILLE ET MISE EN VALEUR DES FERMES

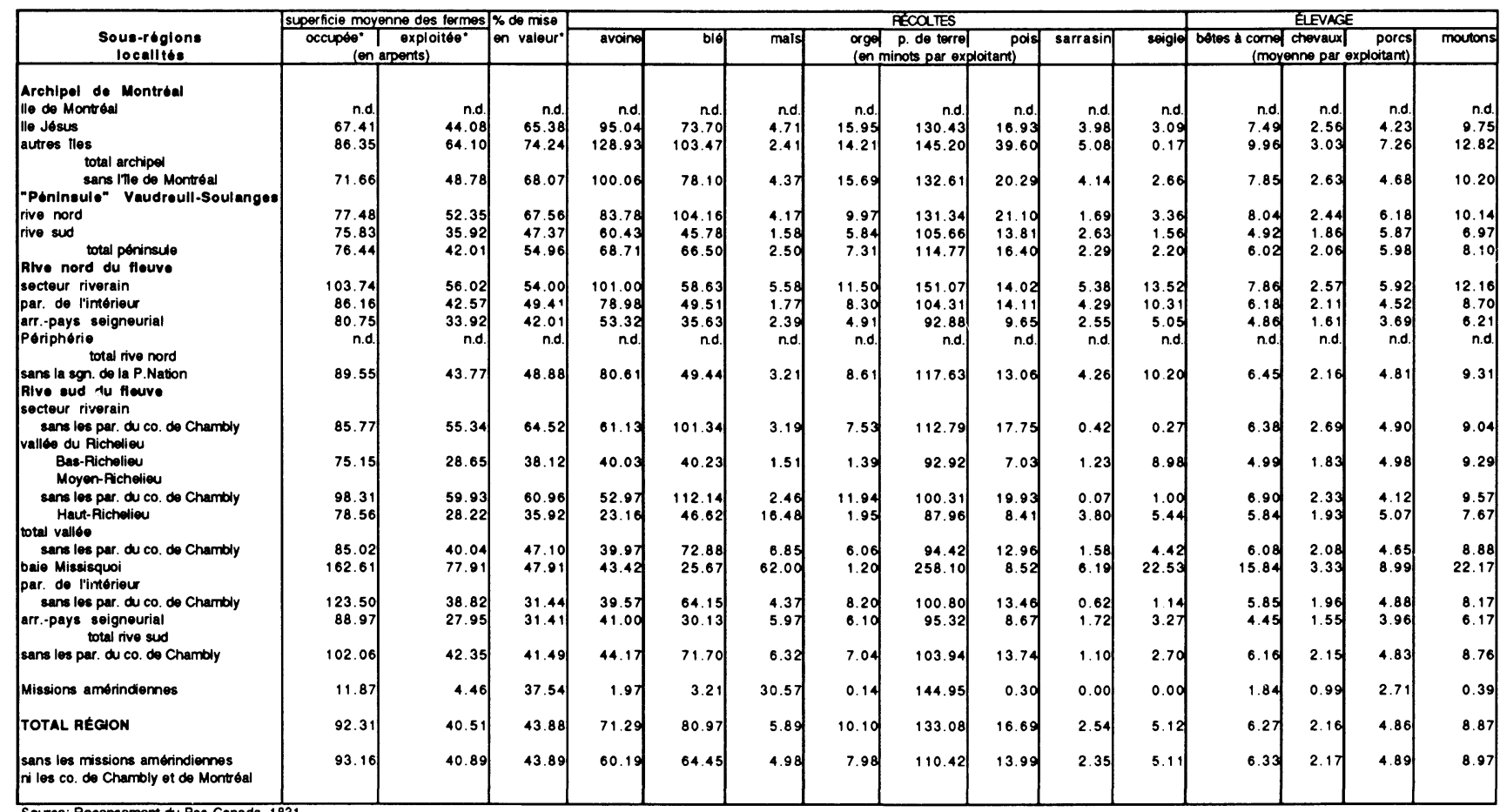


qui relève de l'agriculture de ce qui n'en relève pas. Il arrive cependant que cette distinction ne soit pas toujours possible, lorsque le chef de ménage, par exemple, ne déclare que des animaux, sans récolte pouvant nous permettre d'établir son statut. Le plus souvent, il ne s'agit que de petits cheptels dont la taille est tout juste suffisante pour suggérer une exploitation agricole; mais parfois, il est question d'un cheptel plus important, pouvant même devenir en certains cas démesuré par rapport aux autres qui l'entourent ${ }^{29}$. En tenir compte dans les calculs aurait donc faussé les perspectives. Aussi n'avons-nous retenu comme producteurs agricoles que les chefs de ménage déclarant d'abord des récoltes. Comme la plupart d'entre eux déclarent aussi un élevage, ils regroupent en fait la majorité de ceux qui pratiquent l'agriculture, cumulant même à eux seuls de $80 \%$ à $85 \%$ des troupeaux dénombrés dans le territoire, à l'exception des chevaux dont la part s'établit autour de $77 \%$. Comme tel, le volume des récoltes n'a pas été un facteur discriminant, sauf peut-être dans le cas des très petites productions et encore, puisque c'est l'ensemble de l'agriculture ici qu'il nous intéressait d'observer, peu importent la taille des exploitations, le métier déclaré par les chefs de ménage ou leur lieu de résidence: certains cultivateurs produisant plus que les marchands ou les seigneurs, d'autres pratiquant un double métier dont un seul est enregistré au recensement, d'autres encore habitant au village ${ }^{30}$, et d'autres ayant omis de déclarer la superficie de leur ferme ou de leurs champs ou ces informations ayant été mal enregistrées par les commissaires.

C'est donc à l'ensemble des chefs de ménage déclarant des récoltes que nous nous sommes intéressés, quel que soit leur profil individuel. En 1831, on en dénombre près de 25000 , sur les quelque 33000 que comptent les localités au sujet desquelles on dispose d'une information nominative dans les recensements (missions amérindiennes exclues). $\mathrm{Au}$ total, ces ménages réunissent environ 153000 individus sur les 190000 que comptent ces localités. De ce nombre, plus de la moitié habitent la rive sud du fleuve et le tiers, la rive nord. Quant aux maisonnées moyennes, elles gravitent autour de 6 ou 7 personnes, plus élevées dans l'archipel de Montréal et les secteurs riverains que partout ailleurs, en raison d'un plus grand nombre de domestiques (les «serviteurs-fermiers») dans ces secteurs.

\footnotetext{
29 Dans la seigneurie de Blainville, par exemple, le seigneur, Janvier-Domptail Lacroix, déclare posséder à lui seul 1250 moutons, contre une quinzaine en moyenne chez les producteurs de la côte où celui-ci est enregistré. C'est l'un des plus gros troupeaux rencontrés au Bas-Canada.

30 En 1831, le recensement enregistre la totalité des avoirs fonciers et des productions des individus. Beaucoup de chefs de ménage résidant au village y enregistrent donc aussi leur déclaration agraire. Toutefois, il faut bien comprendre qu'une partie seulement de leurs activités agricoles s'y déroulent, l'essentiel ayant pour cadre les côtes ou les paroisses voisines où ces chefs de ménage détiennent aussi des terres, sauf dans certains villages, linéaires notamment, où seul le front de lot est bâti.
} 


\section{Les densités agraires}

Au plan des densités agraires, les contrastes sont plus grands, révélant des écarts parfois considérables entre les sous-régions. Calculées, par exemple, sur la base des superficies moyennes occupées (densités agraires brutes), elles s'élèvent à une quarantaine d'habitants au $\mathrm{km}^{2}$ dans l'archipel de Montréal (50 dans l'île Jésus), à une trentaine sur les rives du fleuve, à 26 dans la «péninsule» Vaudreuil-Soulanges, et à un peu plus de 20 dans les localités de l'intérieur et de l'arrière-pays seigneurial, sauf dans les terroirs neufs de la rive sud où elles s'abaissent à 16 et à 12. Calculées sur la base des superficies exploitées (densités agraires nettes), elles sont plus élevées encore, supérieures à 67 habitants au $\mathrm{km}^{2}$ dans l'archipel de Montréal (presque 82 sur l'île Jésus), à 56 dans les localités riveraines de la rive nord, à 50 dans celles de la rive sud et à 48 dans la «péninsule» Vaudreuil-Soulanges. Dans la vallée du Richelieu, elles varient de 44 à 66 habitants au $\mathrm{km}^{2}$ (78 dans le Bas-Richelieu), pendant que dans l'arrière-pays seigneurial elles s'élèvent à 72 , pour retomber à 27 à l'est de la baie Missisquoi.

\section{Mode de faire-valoir et taille moyenne des fermes}

Au Bas-Canada, la plupart des agriculteurs sont propriétaires du sol qu'ils exploitent. À cet égard, la région de Montréal ne fait pas exception à la règle, plus de $83 \%$ des exploitants agricoles s'y déclarant propriétaires de leurs terres au recensement de 1831, ce pourcentage pouvant même atteindre près de $95 \%$ dans certaines localités de la rive nord du fleuve. Ailleurs, il gravite autour de 79 à 82\%, pour s'abaisser à moins de $70 \%$ dans le Moyen-Richelieu où la propriété bourgeoise paraît plus importante. Quant aux fermes, elles cumulent plus d'arpents sur la rive sud que sur la rive nord, mais sont davantage mises en valeur autour de Montréal, sur les rives du fleuve et dans le Moyen-Richelieu.

En nous basant sur les déclarations disponibles, on peut estimer à 93 arpents environ ( 31,8 hectares) la superficie moyenne des fermes en 1831, un peu moins, 87 arpents, si l'on ne tient pas compte de la propriété villageoise. Dans les terroirs neufs de la rive sud (localités de l'intérieur et de l'arrière-pays seigneurial), elle dépasse 120 et même 160 arpents, pour atteindre environ 98 arpents dans le Moyen-Richelieu. Sur la rive nord, elle gravite autour de 80 à 86 arpents, sauf dans les localités riveraines où elle s'élève à environ 103 arpents. Dans l'archipel de Montréal et la "péninsule» Vaudreuil-Soulanges, elle oscille autour de 70-75 arpents, les plus petites exploitations se retrouvant sur l'île Jésus avec 67 arpents en moyenne. C'est pourtant là et sur la rive nord de la «péninsule» Vaudreuil-Soulanges que la mise en valeur des terres est la plus intense, pouvant atteindre les deux tiers environ de 


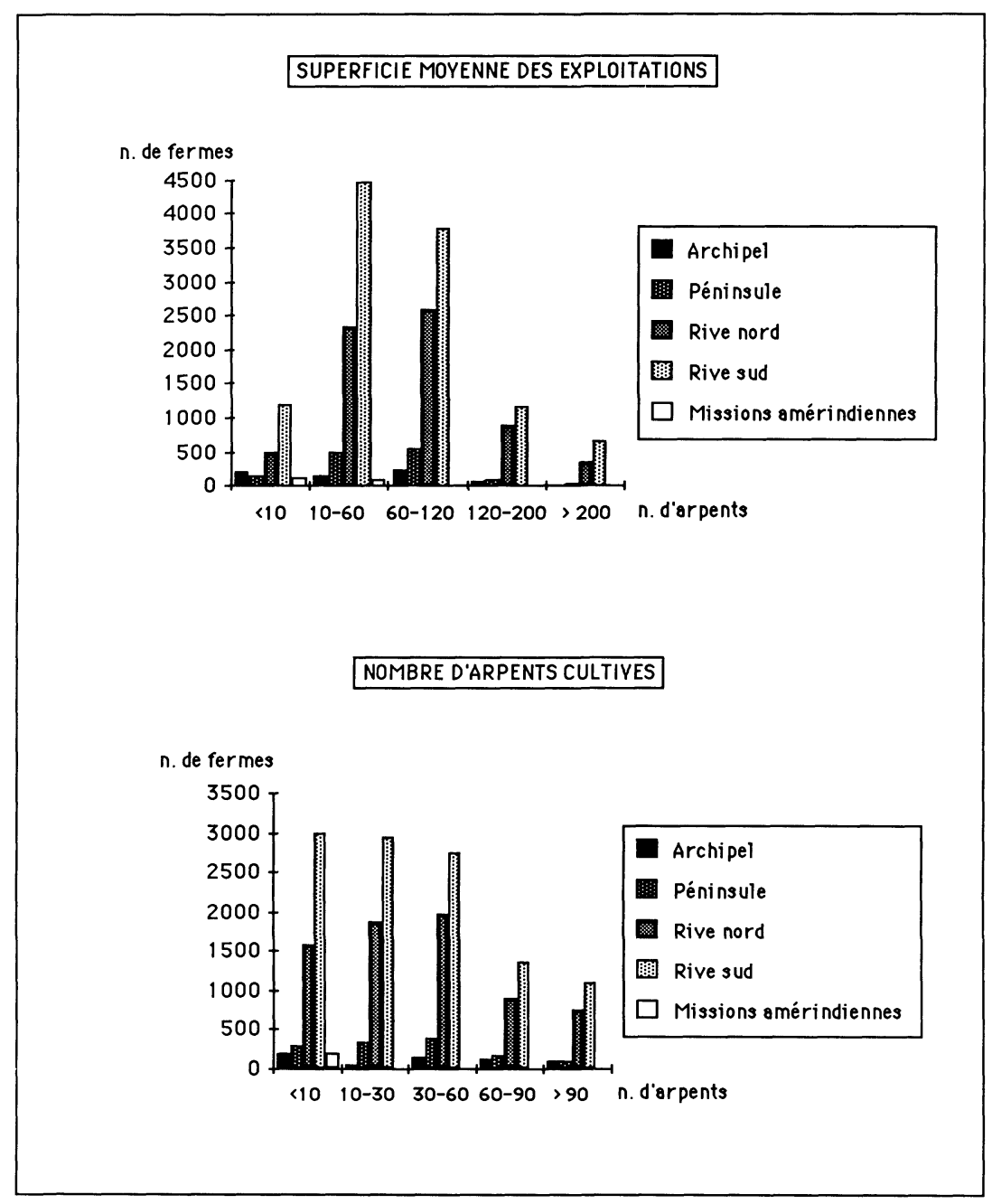

leur superficie totale ${ }^{31}$. Ailleurs, cette proportion s'abaisse au tiers ou à la moitié, sauf sur la rive sud du fleuve et dans le Moyen-Richelieu

31 Au recensement de 1831 , comme à celui de 1842, les superficies déclarées «cultivées» équivalent aux superficies défrichées. Voir Serge Courville, «Origine et évolution des campagnes dans le comté des Deux-Montagnes, 1755-1971», mémoire de maîtrise, Université de Montréal, 1973, 209 p. 
Figure 5

LES EXPLOITATIONS AGRICOLES (1831)

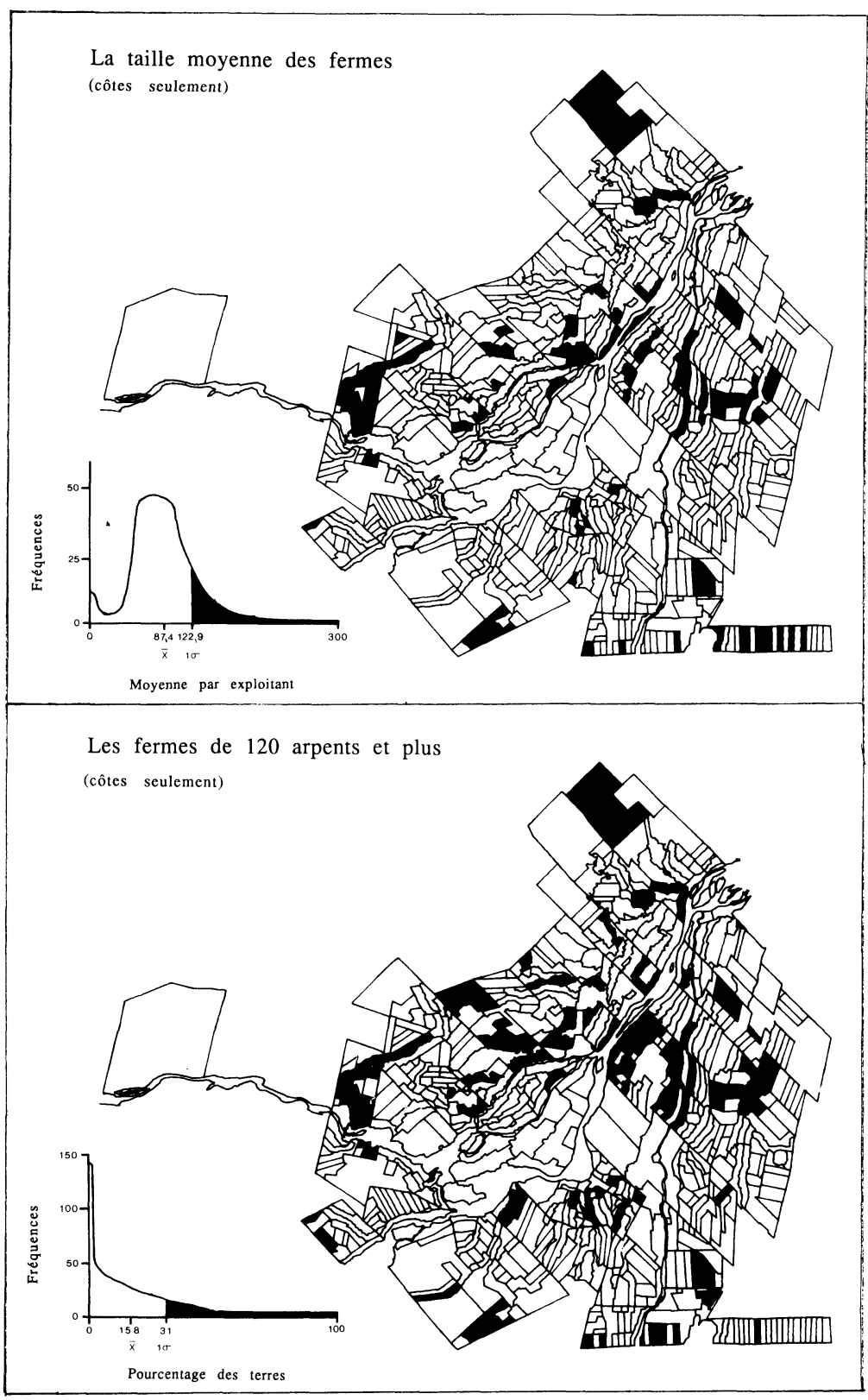


où elle s'élève à plus de $60 \%$. Quant à la structure des exploitations, elle varie considérablement dans l'espace, les plus grandes fermes se retrouvant sur la rive sud du fleuve, tandis que sur la rive nord les fermes de 60 à 120 arpents paraissent proportionnellement plus nombreuses (figures 4 et 5$)^{32}$.

\section{Les productions agricoles}

En 1831, le recensement n'enregistre que les grandes cultures, soit six céréales (blé, orge, avoine, seigle, sarrasin, maïs), les pommes de terre et les pois. C'est donc sur cette base qu'il faut évaluer l'agriculture régionale et ses contrastes, au niveau des grands ensembles d'abord, puis à celui de leurs composantes. L'un des premiers grands constats qu'autorisent les données de recensement est l'écart formidable qui sépare la rive nord et la rive sud du fleuve. Sauf pour les récoltes d'avoine, de sarrasin et de seigle, plus dominantes sur la rive nord du fleuve, les localités de la rive sud produisent entre $40 \%$ et $60 \%$ de la récolte régionale, les plus forts pourcentages allant au blé et au maïs dont environ le cinquième de la récolte régionale provient de la vallée du Richelieu. Les écarts séparant les productions individuelles moyennes sont encore plus prononcés, laissant voir des orientations locales que gomment les pourcentages régionaux: par exemple, le blé, l'avoine, l'orge, le sarrasin et les pommes de terre dans l'archipel de Montréal et sur la rive nord du fleuve; le blé et les pois dans les localités du MoyenRichelieu, certaines îles de l'archipel montréalais et sur la rive nord de la «péninsule» Vaudreuil-Soulanges; le maïs, les pommes de terre, le sarrasin et le seigle sur la baie Missisquoi. Quant à l'élevage, il épouse le même patron, les données totales révélant la suprématie de la rive sud (plus de la moitié des cheptels, quels qu'ils soient), que nuancent localement les données par ferme, notamment sur la rive nord, et dans certaines îles de l'archipel montréalais.

$\mathrm{Vu}$ sous l'angle des productions agricoles, le paysage rural apparaît donc très différencié, plus «agraire» sur la rive sud du fleuve que sur la rive nord et plus intensivement mis en valeur en pays riverain et insulaire que partout ailleurs. Ajoutés aux différences de taille, d'occupation et de mise en valeur des fermes, ses traits d'ensemble rappellent ceux de la plaine allemande à la même époque, où Johann Heinrich von Thünen avait remarqué une organisation en «enveloppes» de l'agriculture ${ }^{33}$. Au centre, dans le voisinage de Montréal et dans l'axe

\footnotetext{
32 Les données représentées sur les cartes par côtes (figures 5 à 9) ne tiennent compte que des valeurs supérieures à un écart-type de la moyenne, comme l'indiquent les graphiques de fréquences insérés au bas des cartes. Cette technique permet de mieux faire ressortir les spécificités locales, qui seraient confondues, autrement, dans les valeurs moyennes.

33 Pour une présentation du modèle de Von Thünen, voir Michael Chisholm, Rural Settlement and Land Use (Londres, Hutchinson University Library, réédition 1970), 20ss, et Peter Haggett, L'analyse spatiale en géographie humaine (Paris, Librairie Armand Colin, 1973), 182ss.
} 
du Richelieu, une zone d'agriculture intensive (et spéculative) où la propriété bourgeoise est importante et où la production des fermes est plus variée; dans son débordement proche, une zone d'agriculture extensive plus tournée vers la production de céréales; puis, dans sa périphérie extrême, une zone d'élevage associé à la culture de pommes de terre.

Toutefois, c'est à l'échelle de la côte surtout qu'il faut observer cette agriculture pour en saisir toute la logique de distribution dans l'espace. On constate alors que les particularités physiques du milieu ne sont pas les seules à exercer une influence sur cette activité. En effet, en n'observant que les côtes qui présentent des avantages similaires sur le plan pédologique (fonds d'argile ou sols argilo-limoneux des petites vallées-plaines), on constate que l'agriculture y est parfois très différente, ses profils étant ceux d'une activité qui, tout en bénéficiant de conditions de culture et d'élevage très semblables, choisit de s'orienter délibérément vers d'autres combinaisons plus en rapport avec les conditions économiques locales. Parmi ces conditions, il y a d'abord celles qu'introduit la présence d'un village ou d'un gros bourg urbain, dont la taille est suffisante parfois pour représenter un marché non négligeable, puis toutes celles qu'introduisent les industries rurales, dont plusieurs sont également un débouché pour l'agriculture locale.

\section{LES SYMBIOSES AGRICULTURE-INDUSTRIE}

Plusieurs facteurs peuvent expliquer les transformations de l'agriculture depuis la fin du 18e siècle: la croissance démographique, la montée de la population urbaine, la poussée villageoise et aussi la montée de l'industrie rurale qui, par la variété même de ses besoins, favorise une diversification de la production. Dans quelle mesure l'industrie estelle responsable de ce changement? On l'ignore encore. Toutefois, à en juger par certaines orientations locales, son rôle semble indéniable, différent sans doute de celui que joue le blé dans le commerce international, mais suffisant pour entraîner une certaine commercialisation des fermes. Ajouté à tous les débouchés qu'offrent le marché villageois et le marché citadin, ce facteur semble même avoir favorisé le passage d'une agriculture de subsistance vers une agriculture plus spéculative, où la satisfaction des besoins domestiques reste importante, mais où l'on cherche aussi à produire des excédents commercialisables réguliers. C'est la caractéristique essentielle d'une agriculture dite de marché. En 1831 , cette agriculture reste très localisée dans l'espace ${ }^{34}$. Elle cor-

\footnotetext{
Voir aussi Serge Courville, «La crise agricole du Bas-Canada, éléments d'une réflexion géographique (première partie)», Cahiers de géographie du Québec, 24,62 (1980): 205ss.

Dans sa thèse, par exemple, Allan Greer a bien montré quelle distance séparait, sur le plan agricole, des paroisses comme Saint-Denis, Saint-Ours et Sorel. Sans conclure à l'existence d'une agriculture commerciale, il observe qu'à Saint-Denis, où les sols sont plus favorables, les cultivateurs sont nettement plus prospères, pendant qu'à Sorel ils le sont beaucoup moins, l'agri-
} 
respond aux aires de distribution des villages et des industries rurales, dont la coïncidence avec les bons sols est frappante. Tout cela suggère des rapports très étroits entre l'agriculture et l'industrie, d'autant plus que cette dernière à l'époque ne répond pas qu'à des besoins locaux: elle répond aussi à des besoins extérieurs, le marché urbain notamment et, en certains cas, le marché international ${ }^{35}$. Elle apparaît donc susceptible d'influences multiples sur l'agriculture.

Pour connaître ces influences, il faudrait pouvoir dépouiller les contrats ou les listes d'approvisionnement de chacune des entreprises retrouvées dans ces aires. Nos travaux n'en sont pas encore là, d'autant moins qu'aux difficultés de retrouver ces documents s'ajoute ici celle de leur disponibilité à l'échelle régionale. Tout ce que l'on peut en dire pour l'instant se résume aux traces qu'ont pu laisser dans le paysage les rapports agriculture-industrie, rapports d'approvisionnement d'abord, puis rapports de main-d'oeuvre.

\section{L'approvisionnement en matières premières}

Le premier constat autorisé par la cartographie des données de recensement concerne les lieux de répartition des industries rurales dans l'espace régional: loin d'être aléatoire, leur distribution correspond aux aires où s'affirme une certaine spécialisation agricole. C'est le cas, entre autres, des moulins à farine, partout présents dans le territoire, mais davantage sur la rive sud du fleuve, dans la vallée du Richelieu notamment, où domine la culture du blé. C'est le cas, aussi, des tanneries et des moulins à fouler et à carder également très répandus dans l'espace, mais davantage dans les terroirs où les élevages bovin et ovin sont importants. C'est le cas également des brasseries et des distilleries dont la répartition semble obéir aux mêmes incitatifs, mais tributaire cette fois des terroirs consacrés à l'orge et au seigle. Enfin, c'est le cas des scieries et des fabriques de potasse, abondantes sur les fronts pionniers et là où les fermes comprennent un pourcentage élevé de boisés. Autrement dit, contrairement à beaucoup d'industries apparues plus tard au cours du siècle et qui rechercheront plutôt le marché, l'industrie rurale à l'époque recherche surtout les matières premières, c'est-à-dire les lieux de production où elle peut bénéficier d'un approvisionnement sûr et abondant, en plus d'une disponibilité de main-d'oeuvre.

En soi, cette répartition est en quelque sorte «normale», expliquant surtout le comportement de l'industrie. Pour saisir celui de l'agricul-

culture ici cédant la place à une industrialisation qu'accompagnent un abaissement de l'âge des hommes au mariage et un redressement du bilan migratoire. Allan Greer, Peasant, Lord and Merchant. Rural Society in Three Quebec Parishes (Toronto, Buffalo, London, University of Toronto Press, 1985), 227ss.

35 C'est le cas, entre autres, de la farine, du bois d'oeuvre et de la potasse, acheminés en partie sur le marché intérieur mais en partie sur le marché extérieur, à destination de l'Angleterre notamment. 
ture, il faut changer d'échelle et observer le phénomène localement. C'est ce que nous avons fait, en cartographiant les données par côtes. Ce qui ressort de cette cartographie est révélateur des avantages dont bénéficie l'agriculture locale quand elle dispose d'un débouché à proximité. En effet, loin d'être uniformément distribués dans l'espace, les cultures et les élevages à fonction industrielle ont tendance à augmenter dans le voisinage des lieux où se répartissent les industries rurales, les côtes les plus productrices étant celles qui accueillent de telles industries, ou les côtes voisines si les conditions de sols y sont plus favorables (figures 6, 7 et 8). Ici, c'est donc la présence d'industries qui explique les spécialisations locales. On en a un exemple dans la production d'orge et de seigle, toujours plus importante là où l'on enregistre la présence locale de brasseurs (le village de Saint-Eustache, par exemple, sur la rive nord, ou ceux de Laprairie ou de Saint-Hyacinthe sur la rive sud), ou dans les secteurs de répartition des distilleries, le cas le plus significatif à cet égard demeurant celui de l'archipel montréalais et de la rive nord du fleuve où l'on ne dénombre pas moins de sept distilleries qu'approvisionne une aire vaste de production allant de la seigneurie de la Rivière-du-Chêne à la seigneurie de Lavaltrie en passant par celle de l'île Jésus et de l'île de Montréal, et sans doute aussi en partie, à l'aval, les seigneuries de Lanoraie, Dautré et Berthier, grâce aux chemins qui unissent ce secteur aux précédents. Dans ce dernier cas, toutefois, il n'est pas impossible qu'une partie de la production soit écoulée aussi vers la distillerie de Contrecoeur, située sur la rive sud du fleuve. On observe un phénomène similaire avec la pomme de terre, dont on sait la place dans l'alimentation domestique, et celle des porcs, mais dont la récolte, toujours plus élevée dans ces secteurs, semble également alimenter un marché, peut-être destiné aux distilleries locales. Même tendance, enfin, avec l'élevage ovin, toujours plus important dans le voisinage des côtes où se retrouvent des moulins à fouler ou à carder, les secteurs les plus représentatifs à cet égard demeurant, sur la rive nord, celui de la rivière Bayonne (paroisse de Berthier), celui de la rivière l'Assomption et de ses affluents, celui de la rivière Mascouche, et celui de la rivière du Nord, et, sur la rive sud, celui de la rivière Châteauguay, ceux de la rivière Richelieu (secteurs de Saint-Ours et de Saint-Jean) et de la rivière Yamaska (secteur de Saint-Hyacinthe), et les environs du Mont-Saint-Hilaire. Autrement dit, ce qui aurait dû être caractéristique des fronts pionniers (céréales pauvres, élevage ovin) le devient plutôt des secteurs les plus densément occupés et les plus riches sur le plan pédologique. Bien plus, c'est aussi là, dans ces secteurs (et dans ceux de fortes récoltes en blé) que la superficie des exploitations est la plus grande et que la mise en valeur des terres est la plus intense, comme si la présence d'industries se conjuguait ici avec le marché pour commander des combinaisons agricoles spécifiques, distinctes de celles retrouvées dans les côtes ou les localités voisines où l'agriculture semble correspondre davantage au type d'agriculture décrit par la littérature 
Figure 6

\section{LA CULTURE DE L'ORGE (1831)}

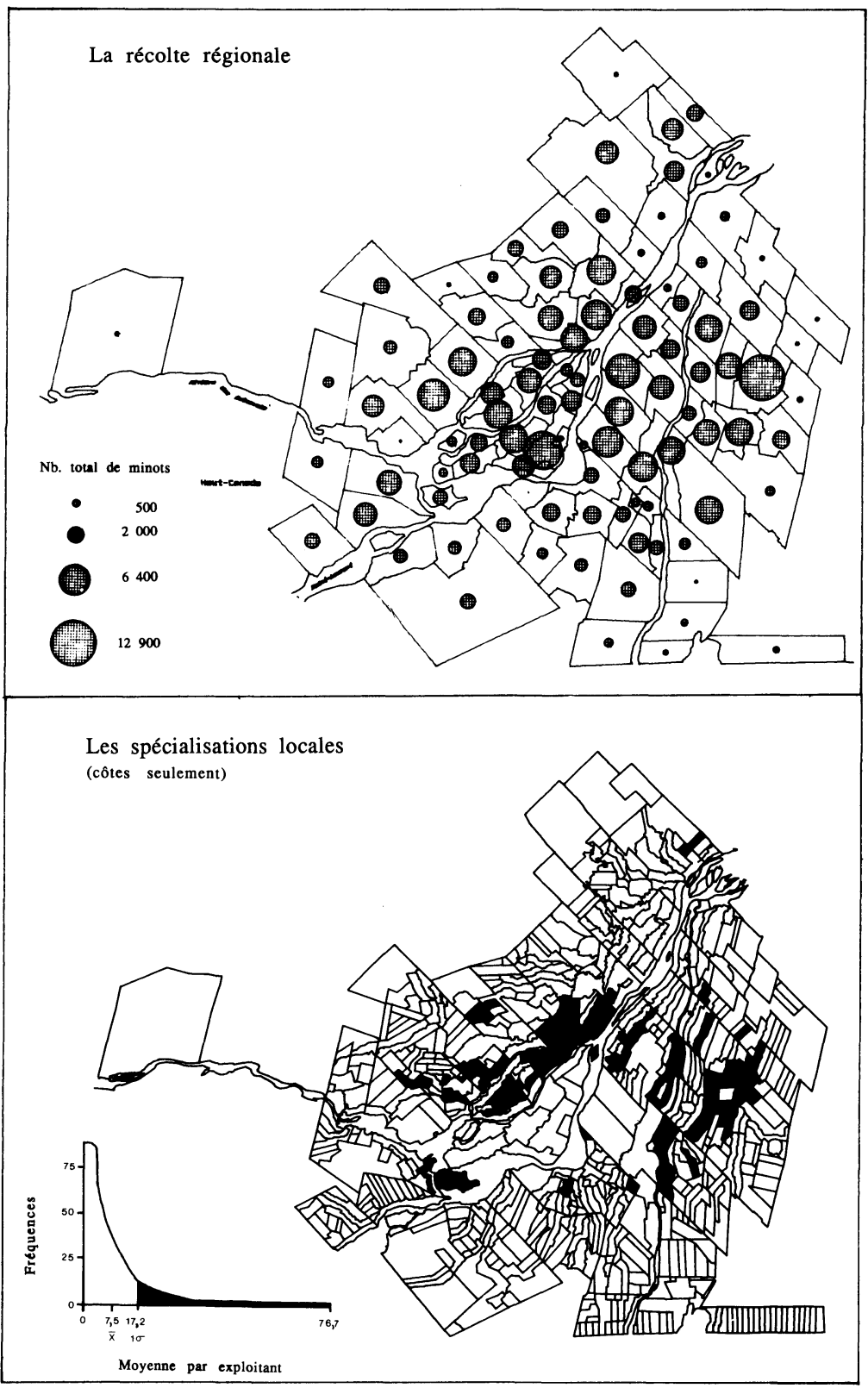




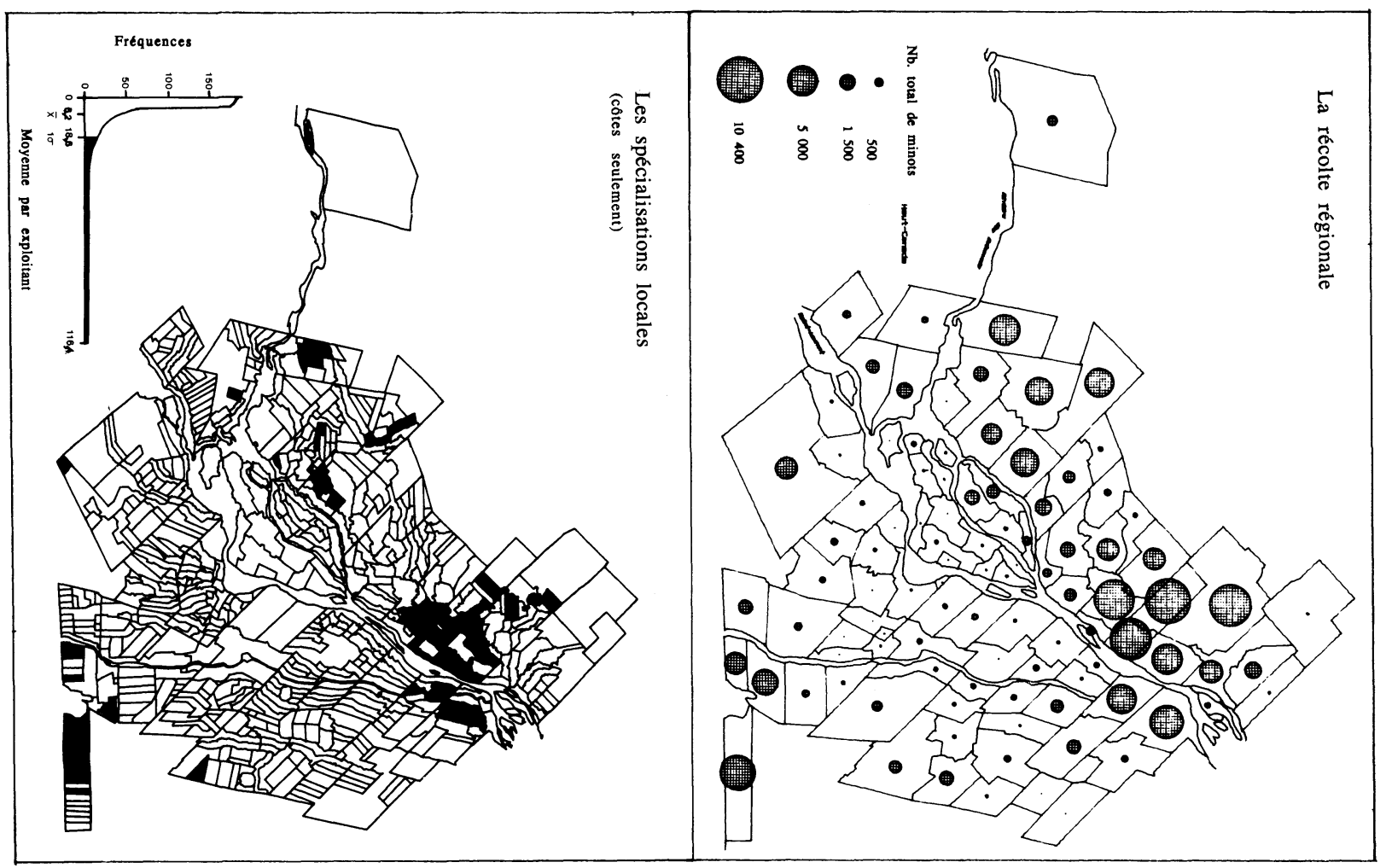

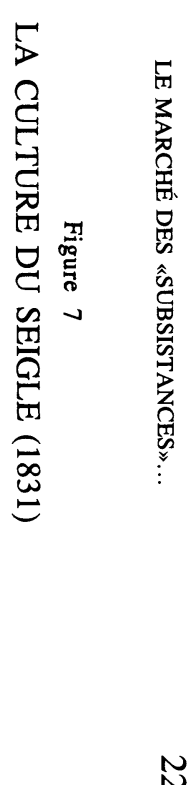


Figure 8

L'ÉLEVAGE OVIN (1831)

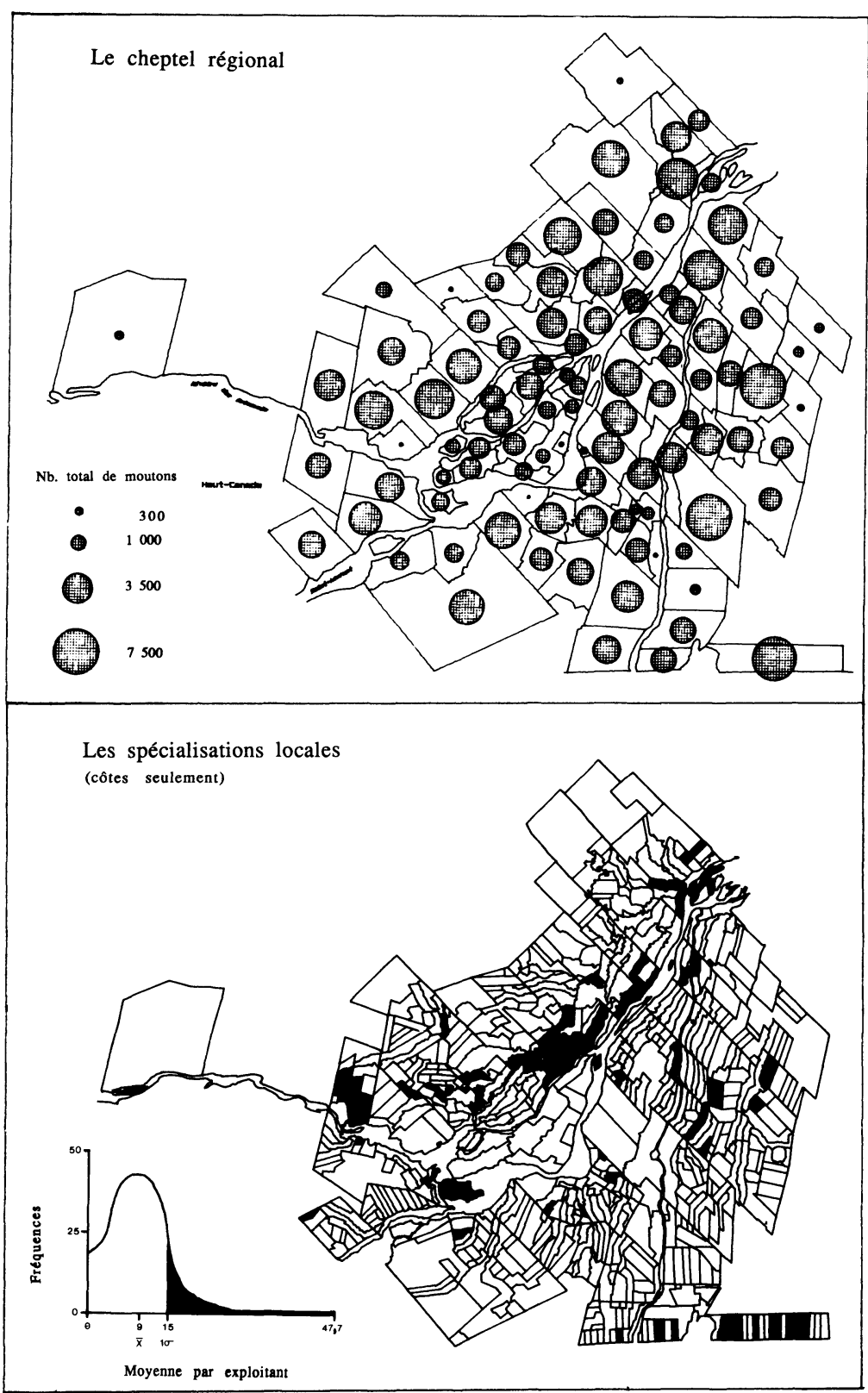


scientifique. Tout cela évoque, encore là, le patron de Von Thünen. Mais il n'y a pas que l'agriculture qui soit ainsi influencée par la présence d'industries. La main-d'oeuvre l'est également.

\section{L'attrait sur la main-d'oeuvre}

Parmi les facteurs qui expliquent la prolifération des industries rurales et leur diffusion dans l'espace, il y a l'abondance de la maind'oeuvre, partout disponible, tant au village que dans les côtes. Manque-t-elle, qu'on l'importe, ce qui est le cas, entre autres, sur certains fronts pionniers ou dans certaines seigneuries où progresse l'exploitation forestière ${ }^{36}$. Une fois implantée, toutefois, l'industrie attire même les producteurs agricoles qui trouvent là des revenus d'appoint non négligeables ou qui en font l'objet d'un travail plus permanent, abandonnant leur exploitation aux autres membres du ménage (enfants et engagés).

En effet, en ne retenant que les chefs de ménage pouvant être considérés comme «producteurs agricoles» au recensement, on constate que nombreux sont ceux qui semblent avoir préféré l'industrie à l'agriculture ou du moins entretenir des rapports très étroits avec elle (figure 9). C'est le cas, entre autres, de tous ceux qui déclarent un métier relatif au textile: on ne les retrouve, pour l'essentiel, que dants le voisinage des moulins à fouler et des moulins à carder. C'est le cas aussi de tous ceux qui déclarent un métier relié au travail du cuir et à la fabrication d'alcool: ils sont à peu près tous localisés dans le voisinage des tanneries (dont le nombre est sans doute supérieur à celui mentionné par Bouchette) et dans celui des brasseries et des distilleries. Quant aux journaliers, en nombre croissant à l'époque, ils semblent aussi très nombreux à entretenir des rapports avec l'industrie, dont ils recherchent très souvent le voisinage. C'est le cas, par exemple à Saint-Eustache où nous avons déjà montré leur répartition dans l'espace ${ }^{37}$. C'est le cas aussi dans certaines localités de la rive sud, au deuxième rang de SaintCharles par exemple, où l'on enregistre une forte proportion de journaliers à proximité des six moulins à farine et des cinq moulins à scie du premier rang (plus du quart des chefs de ménage retenus comme producteurs dans nos listes, sans compter tous les autres situés dans les côtes voisines ou ne déclarant pas de productions agricoles). Le même phénomène peut être observé également dans le voisinage des moulins à scie et du moulin à farine de la Grande-Côte de Lanoraie, où plus du

\footnotetext{
36 On en a un exemple dans le cas de Joseph Papineau qui, pour exploiter sa seigneurie de la Petite Nation, fait venir à grands frais des colons et ce, dès la fin des années 1810 . On en a un autre dans le cas du seigneur Joly de Lotbinière qui va même jusqu'à aménager un véritable petit «quartier ouvrier» à proximité de son moulin de Leclercville.

37 Serge Courville, «Croissance villageoise et industries rurales [...]», François Lebrun et Normand Séguin, Sociétés villageoises et rapports villes-campagnes [...], 205-219.
} 
Figure 9

LES RAPPORTS DE MAIN-D'OEUVRE (1831)

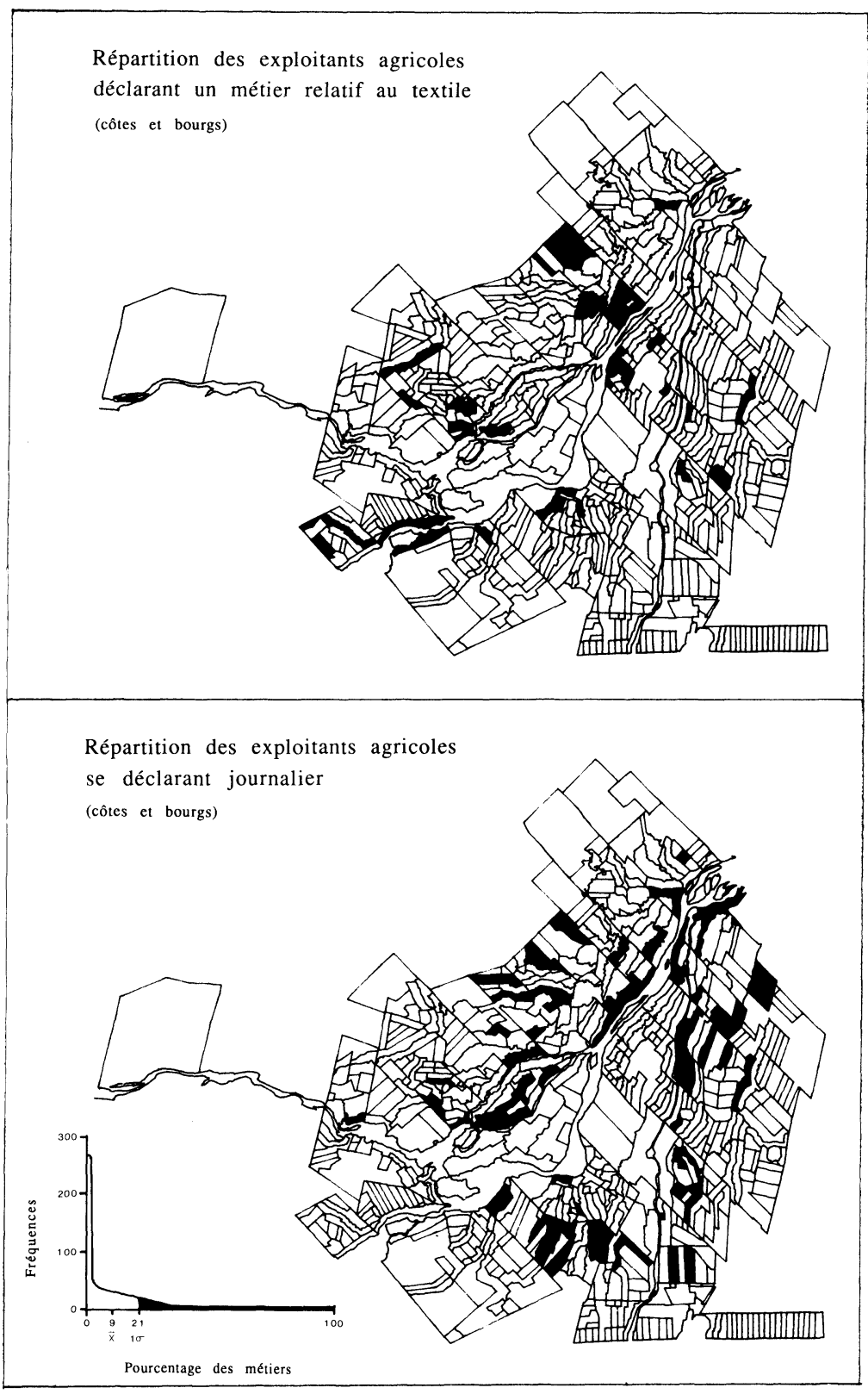


tiers des producteurs de la côte voisine (concession de la rivière SaintJean) se déclarent journaliers. Enfin, la même situation prévaut dans le voisinage des moulins de la rivière Mascouche où cette fois le nombre de journaliers s'élève à plus de la moitié des chefs de ménage enregistrés comme producteurs dans nos listes.

Cela ne signifie pas que les journaliers n'ont de rapports qu'avec l'industrie, plusieurs d'entre eux pouvant très bien oeuvrer dans d'autres domaines d'activités, incluant celui de l'agriculture. Toutefois, comme ils sont déjà eux-mêmes producteurs, on peut se demander dans quelle mesure ils s'engagent comme ouvriers agricoles? Il y aurait peut-être une distinction à faire ici entre les journaliers-producteurs pour qui la pluriactivité, selon le mot d'Yves Rinaudo ${ }^{38}$, s'exprime plutôt dans le travail artisanal ou industriel, et les journaliers non producteurs peutêtre plus susceptibles de s'engager comme ouvriers agricoles, chacun pouvant aussi, bien sûr, trouver de l'emploi chez un cultivateur voisin, dans l'entretien, le bâtiment, le transport ou les services. L'hypothèse est d'autant plus plausible que dans les secteurs de faible industrie rurale la part de journaliers s'abaisse sensiblement, au profit des métiers relatifs à l'agriculture (cultivateurs, fermiers, laboureurs). Quoi qu'il en soit, ce que l'on sent surtout à travers cette présence accrue de journaliers-producteurs dans les secteurs riches en industries rurales, c'est tout le passage de l'agriculture à l'industrie que sanctionne la faiblesse de leurs récoltes ou de leur élevage (quelques minots de céréales ou de pommes de terre, un cheval ou deux, quelques têtes de bétail, un petit élevage de porcs ou de moutons, sauf dans certains cas particuliers ou dans ceux des journaliers qui travaillent pour quelqu'un d'autre) et que vient confirmer la diversité de métiers déclarés par les producteurs agricoles. Pour la plupart d'entre eux, particulièrement nombreux chez les francophones, l'agriculture reste une activité secondaire pratiquée dans l'attente d'un travail salarié. Il ne faudrait pas en conclure, toutefois, que tous les producteurs francophones soient dans ce cas, ou que leur agriculture soit moins performante. La réalité est beaucoup plus complexe.

\section{Le facteur ethnique}

Dans les études sur l'agriculture au Bas-Canada, on a souvent invoqué les différences ethniques comme facteur principal de différenciation des campagnes, les anglophones paraissant faire preuve de meilleures techniques dont la diffusion tarde chez les francophones et ce, même dans les secteurs proches des villes ${ }^{39}$. Important, ce facteur n'ex-

\footnotetext{
38 Yves Rinaudo, «Un travail en plus: les paysans d'un métier à l'autre (vers 1830 - vers 1950)», Annales ESC, 42,2 (mars-avril 1987): 283-302.

39 Voir Jean-Claude Robert, «Activités agricoles et urbanisation dans la paroisse de Montréal, 1820-1840», François Lebrun et Normand Séguin, Sociétés villageoises et rapports villescampagnes $[. .],. 91-100$.
} 
plique pas tout, d'autant moins que dans les aires de forte symbiose agriculture-industrie, le gros de la population agricole est d'origine francophone et que les ménages d'origine étrangère qu'elle côtoie sont souvent des voisins avec qui elle entretient des liens très divers ${ }^{40}$. En outre, comme les bassins d'approvisionnement des industries rurales sont parfois très larges, ils rejoignent quantité de producteurs dont plusieurs, sinon la majorité, sont des francophones. Enfin, même dans les secteurs d'agriculture anglophone, les liens avec l'extérieur sont nombreux, touchant encore là quantité de francophones dont plusieurs s'adonnent à des cultures ou des élevages destinés à l'industrie.

À Saint-Eustache, par exemple, où l'on retrouve un brasseur d'origine anglophone établi au village (John Welsteed), les marchands William Scott et Ed Colls déclarent respectivement 100 et 60 minots d'orge auxquels s'ajoutent les 70 minots du yeoman McLoad. Pourtant, les plus gros producteurs sont des cultivateurs francophones établis dans les côtes voisines: F.-X. Grignon du Petit-Brûlé, par exemple, déclare 200 minots, Jean-Baptiste Fortier, son voisin, 130, Joseph Paiement de la Grande-Fresnière, 100, et Joseph Grenier du Grand-Chicot, 106. Quant aux côtes les plus productrices, elles sont toutes voisines du village: Grande-Côte, Petite-Rivière-Nord, Grande-Fresnière, PetitBrûlé, etc. On observe le même phénomène à Sainte-Thérèse, où la production des anglophones est en général plus élevée et où les plus gros producteurs sont le marchand James Porteous et ses employés, mais où la production de certains cultivateurs francophones établis non loin du village est loin d'être négligeable. Même phénomène également dans les seigneuries d'Argenteuil et de Beauharnois, où les anglophones sont nombreux mais où ils ne sont pas les seuls à produire pour le marché. Ailleurs, les écarts sont plus grands, à l'avantage tantôt des uns, tantôt des autres, sauf dans le Haut-Richelieu et la seigneurie de Saint-Armand où les francophones étant peu nombreux le lien agriculture-industries rurales reste surtout une affaire d'anglophones. Toutefois, à considérer l'importance relative de certaines récoltes dans ces derniers secteurs, on peut se demander si les aires d'approvisionnement de certaines industries locales (les distilleries notamment) ne sont pas plus larges, étendues aux cantons ou aux États-Unis voisins et peut-être même aux localités du Moyen-Richelieu?

Tout cela suggère une importante vie de relation, d'autant plus animée que certaines côtes, pourtant privées d'industries rurales, produisent plus que la moyenne locale et, pour certaines, régionale. C'est le cas, entre autres, de la production de blé, de laine, de cuir, de cendres pour la potasse, dont l'aire d'extension déborde largement les secteurs

40 La distinction entre francophones et anglophones ou autres a été établie dans nos travaux à partir d'une approche patronymique plutôt que sur la base des dénominations religieuses enregistrées au recensement, qui ne distinguent pas les catholiques anglophones. 
immédiats de localisation des industries correspondantes et touche une masse imposante de producteurs, tant francophones qu'anglophones. Aussi n'est-ce pas tant le facteur ethnique ici qui importe, que la présence elle-même d'industries, qui, par les débouchés offerts, attire un nombre de plus en plus important de producteurs, quelle qu'en soit l'origine. Que ces industries appartiennent ou non à des intérêts étrangers ${ }^{41}$ ne change rien au fait qu'elles représentent un marché à satisfaire, dont l'importance croît avec celle de ces entreprises ellesmêmes. Mais plus que leur taille individuelle, c'est surtout leur nombre et leur densité dans l'espace qu'il faut prendre en compte, ces facteurs contribuant, avec le village, à définir le contour des aires où s'activent les échanges.

\section{Un cas de «proto-industrialisation»?}

Tout ce qui précède nous conduit naturellement à l'idée qu'il a pu exister au Bas-Canada un contexte de croissance semblable à celui observé dans d'autres contrées du monde où les orientations de l'agriculture semblent avoir été très influencées par l'évolution du marché et la montée des industries rurales. Prétendre à un tel contexte au BasCanada relève encore de l'hypothèse, d'abord parce que trop de données font défaut, notamment sur le type de fabrication qu'encourage la ville, sur les types de marchés où s'écoulent ces fabrications, sur le rôle des marchands (et de leurs intermédiaires) dans la mise en place des organisations de production, sur l'importance du travail domestique, etc. En outre, rien ici n'a l'ampleur des phénomènes observés ailleurs dans le monde; au contraire, tout est à la mesure d'une province qui ne compte encore qu'un peu plus de 500000 habitants après 200 ans d'histoire. Enfin, parmi les industries rurales recensées, nombreuses sont celles encore qui, en 1831, n'ont encore qu'une fonction locale, assez semblable somme toute à celles qu'elles avaient autrefois en contexte européen ${ }^{42}$. Pourtant, des phénomènes très similaires se produisent, qui suggèrent d'élargir la problématique de l'agriculture au Bas-Canada, en regard peut-être de l'hypothèse élaborée par Franklin F. Mendels sur les prémisses de la révolution industrielle en Europe ${ }^{43}$.

41 Nous ne disposons pas encore de relevé exhaustif de propriétaires d'entreprises en 1831. Toutefois, si les brasseries et les distilleries semblent surtout appartenir à des intérêts anglophones, les moulins à farine, les moulins à fouler et les moulins à carder, ainsi que les moulins à scie semblent plutôt appartenir à des intérêts francophones, sauf dans les secteurs anglophones et dans les cas de très grandes exploitations.

42 C'est le cas, entre autres, de bien des petits moulins à farine et à scie dont l'origine remonte parfois au tout début des établissements locaux et dont la capacité unitaire de production reste bien en deçà de celle des gros moulins et de ceux qui seront alimentés plus tard par la vapeur: leur insertion dans les circuits du marché est donc le plus souvent ponctuelle et fonction des surplus saisonniers. Toutefois, cela ne veut pas dire qu'ils ne satisfont que des besoins locaux: il faut prendre en compte ici leur nombre et leur capacité globale de production, d'autant plus qu'après 1815-1820, on assiste à une réorientation générale de l'agriculture qui en accroît la densité dans l'espace.

${ }_{43}$ Cette hypothèse a donné lieu à plusieurs thèses dont certaines très formalisées. Ce sont moins ces thèses qui nous intéressent ici que la notion même de proto-industrialisation. Cette 
En effet, si, à l'instar de cet auteur, l'on définit la proto-industrialisation comme «la transformation structurelle d'une région dont la paysannerie travaille de plus en plus à la production artisanale de biens manufacturés destinés au marché extra-local, tandis qu'une agriculture commerciale se développe simultanément par ailleurs dans une région proche» ${ }^{44}$, il faut admettre que des indices sérieux existent en ce sens, qui ne définissent peut-être pas une situation "proto-industrielle» au sens où Mendels l'entend, mais qui, toutes nuances gardées, s'en rapproche suffisamment pour qu'il vaille la peine de poser le problème. Sachant l'importance que celui-ci accorde à la notion de région polarisée et à tous les contrastes qui la caractérisent en situation proto-industrielle, on peut se demander ce qu'il en est dans celle de Montréal où existe une importante ville-marché, où les débouchés industriels sont nombreux et où apparaîtra bientôt tout un réseau de petites villes manufacturières greffé à l'ancien réseau villageois et aux principaux secteurs de localisation des industries rurales. Par exemple, on y retrouve la divergence écologique invoquée par Mendels dans l'élaboration de son hypothèse, la rive sud du fleuve paraissant beaucoup plus avantagée sur le plan du relief et des sols que la rive nord. On y retrouve aussi ce partage entre une rive sud céréalière, presque entièrement consacrée au blé et tournée vers le marché extérieur, et une rive nord plus vivrière, davantage tournée vers le marché intérieur, où l'élevage abonde, où les industries rurales sont nombreuses et où le travail industriel est très diversifié. Ce que nos travaux, en outre, ont montré, ce sont tous les contrastes retrouvés à l'intérieur même de ces vastes sous-ensembles entre les secteurs d'agriculture plus spéculative et les secteurs plus tournés vers la satisfaction des besoins domestiques où, dans un cas, et en dépit de densités rurales plus élevées et d'un nombre parfois notable de petites fermes, les exploitations sont plus grandes, la propriété bourgeoise plus importante, les maisonnées plus faibles et les engagés agricoles plus nombreux, et où, dans l'autre, les fermes sont plus petites, les densités agraires plus élevées et le nombre d'engagés plus restreint,

notion, très riche, peut être utile dans l'étude du Bas-Canada. Elle renvoie à toute une série de considérations dont on trouvera une présentation dans Serge Courville, «Un monde rural en mutation: le Bas-Canada dans la première moitié du XIXe siècle», à paraître dans Histoire sociale/ Social History, 1988.

44 Franklin F. Mendels, «Aux origines de la proto-industrialisation», Bulletin du Centre d'histoire économique et sociale de la région lyonnaise, 2 (1978): 2. Pour une présentation plus complète de l'hypothèse de Mendels et de ses conditions, voir Franklin F. Mendels, Industrialization and Population Pressure in Eighteenth-Century Flanders (New York, Arno Press, 1981), 312 p.; «Seasons and regions in agriculture and industry during the process of industrialization», Sidney Pollard, ed., Regions and Industrialization. Studies in the Role of the Region in the Economic History of the Last Two Centuries (Gottingen, 1980), 177-195; «Proto-Industrialization: the First Phase of the Industrialization Process», The Journal of Economic History, 32 (1972): 241-261; «Les temps de l'industrie et les temps de l'agriculture. Logique d'une analyse régionale de la proto-industrialisation», Revue du Nord, 63,248 (1981): 21-33; et «Des industries rurales à la proto-industrialisation: historique d'un changement de perspective», Annales, 39,5 (1984): 977 1008 . 
les secteurs les plus avantagés sur le plan agricole demeurant ceux de l'archipel de Montréal, des rives immédiates du fleuve et de ses principaux affluents, et des petites vallées-plaines riches en villages et en industries. Ailleurs, l'agriculture semble moins spéculative, encore que la superficie moyenne des fermes y reste respectable. Mais comme on $\mathrm{y}$ trouve aussi quantité de journaliers et très peu d'industries, il est possible que ces autres secteurs servent en réalité de bassin de maind'oeuvre pour les secteurs plus avantagés, qui y puiseraient au gré des besoins et de la conjoncture.

Loin d'être une source de tensions à l'intérieur de la région, ces contrastes semblent au contraire l'unifier, devenant un facteur d'échange important à l'intérieur du territoire, dont témoignent les courants migratoires intra-régionaux ${ }^{45}$, les spécialisations locales de la main-d'oeuvre, l'aire d'extension de certaines cultures ou de certains élevages autour des lieux de concentration des villages et des industries rurales, et le rôle que semblent jouer les entreprises locales dans les vocations de certaines côtes ou de certaines exploitations, même quand celles-ci sont situées à distance des lieux immédiats de localisation des moulins ou des autres fabriques. En effet, par delà les contrastes régionaux, ce qui frappe finalement dans le paysage rural du début des années 1830 , ce sont les équilibres que semblent introduire localement cette compénétration de côtes plus productrices et de côtes qui le sont moins ou très peu et, à l'intérieur de celles-ci, cette coexistence de petites et de grandes fermes où, les orientations de l'une n'étant pas nécessairement celles de l'autre, les productions de chacune peuvent devenir complémentaires $^{46}$, créant ainsi des conditions favorables à la vie d'échange.

Dans notre exposé, nous ne nous sommes intéressé qu'à la population rurale agricole, c'est-à-dire aux chefs de ménage pouvant être considérés comme producteurs agricoles, à leur famille et à leurs engagés. Il y a aussi tous les autres, particulièrement nombreux sur la rive nord du fleuve. En 1830, la plupart sont artisans ou trouvent de l'emploi dans les industries locales, le bâtiment et les services, ce qui

\footnotetext{
45 En 1851-1852, par exemple, près de la moitié de la population dénombrée dans les bourgs de la région de Montréal déclare un lieu d'origine situé hors de la localité recensée, les secteurs les plus «ouverts» à l'immigration demeurant ceux de la partie nord de la région, incluant l'archipel de Montréal et la «péninsule» Vaudreuil-Soulanges. Même en défalquant les personnes de moins de 20 ans, les chiffres restent impressionnants.

46 Le recensement de 1831 n'enregistre pas les productions de laine ou d'étoffe des ménages. Par contre, on sait qu'elles furent importantes. En 1827, par exemple, on évalue à plus de 1,5 millions d'aunes de France la fabrication de l'étoffe du pays et à 808240 celle de la petite étoffe. Dans tout le Bas-Canada, on compte alors plus de 13300 métiers à tisser, la plupart actionnés par des femmes. Voir Isidore Lebrun, Tableau statistique et politique des deux Canadas (Paris, Québec, Treuttel et Wurtz, Neilson et Cowan, 1833), 389. En outre, l'on sait par les recensements de 1842 et de 1851 que certains ménages produisaient des étoffes, même sans pratiquer d'élevage ou de culture industrielle, ce qui suggère des circuits d'échange importants entre les exploitations.
} 
permet d'écarter la menace malthusienne. Ce n'est que vers la fin de la décennie que des malaises surgissent, devant la concurrence des industries urbaines dont plusieurs se mettent à l'heure de la machine à vapeur. En outre, même si dans les recensements canadiens on ne peut pas mesurer avec précision la part des membres du ménage occupée au travail rural domestique avant 1851, son importance antérieure ne fait aucun doute, comme en témoigne la quantité de chefs de ménage enregistrés comme fileurs, tisserands, tanneurs ou autres au recensement de 1831 ; il est difficile de croire qu'ils furent les seuls membres du ménage à exercer ces métiers ${ }^{47}$. Enfin, que dire des indices offerts par la superficie moyenne des fermes? Contrairement à ce qu'ont prétendu certains auteurs, très peu d'exploitations véritablement agricoles en 1831 sont subdivisées entre héritiers, leur superficie moyenne étant même supérieure à 90 arpents, ce qui est une norme historique dans la vallée du Saint-Laurent. C'est donc dire que là où des spécialisations agricoles s'affirment il y a aussi d'importants surplus de main-d'oeuvre, dont une partie seulement reste dans l'agriculture. Les autres se dirigent vers le village et les industries locales, $d$ 'autant plus que les bonnes terres se font de plus en plus rares, du moins dans les vieux terroirs, et que l'agriculture est grevée de prélèvements divers (seigneuriaux et autres) qui pèsent lourd sur les jeunes établissements. Il suffit dès lors qu'un marchand, un seigneur ou un capitaine d'industrie réalise ce potentiel, qu'une source d'énergie soit disponible et qu'une demande existe, pour qu'une industrie apparaisse; celle-ci profitera alors des orientations locales de l'agriculture ou les suscitera, offrant des débouchés nouveaux à la ferme et des occasions nouvelles de travail que prolongera le travail rural domestique.

Quant aux conséquences possibles de cette situation sur la démographie locale, elles sont plus difficiles à saisir, le recensement de 1831 ne donnant pas de renseignements sur les naissances et les décès, et regroupant la population par tranches d'âge trop larges pour que l'on puisse en obtenir une vision détaillée. Toutefois, sachant que l'époque considérée en est une de forte croissance démographique et qu'en dépit des pressions exercées sur la terre on cherche à préserver l'intégrité du lot, on peut se demander dans quelle mesure cette symbiose agriculturemarché-industrie a favorisé l'abaissement de l'âge au mariage et influencé le destin socio-professionnel des jeunes couples. À elle seule, cette question nécessiterait une étude en soi, effectuée dans le temps long et dans un territoire assez vaste pour en saisir les nuances. Tout ce que l'on peut en dire pour l'instant se résume à peu de choses, sinon

47 Au recensement de 1851 , par exemple, seulement $45 \%$ des quelque 200000 chefs de ménage dénombrés sont enregistrés comme cultivateurs, les autres sont ou journaliers (ils forment la majorité), ou artisans, ou engagés dans le bâtiment, les services ou le transport, ceci sans compter ceux retrouvés dans l'administration, le commerce ou les professions libérales dont le nombre toutefois est plus restreint. 
que dans une localité où il a été possible d'analyser de plus près les lieux de résidence des chefs de ménage, celle de Saint-Eustache au nord-ouest de Montréal, plus de $98 \%$ des hommes mariés de moins de 30 ans $^{48}$ sont établis dans les secteurs les plus industrialisés de la paroisse (villages de Saint-Eustache et de Saint-Augustin et de leurs environs immédiats, secteurs de la Grande-Fresnière et extrémité est de la GrandeCôte), comme si ces endroits représentaient un lieu d'avenir intéressant, où le travail abonde et où l'agriculture peut bénéficier de meilleurs débouchés. Il n'est pas illusoire de penser que ce phénomène se répète ailleurs dans le territoire, comme le suggèrent le dessin même de nos cartes et les données relatives à certains autres secteurs géographiques ${ }^{49}$. Le phénomène, ici, paraît donc à la fois économique et social, inscrit dans la logique du développement capitaliste de l'époque, mais accordé à un processus de reproduction sociale qui a toujours fait une large place à la mobilité géographique ${ }^{50}$.

Quoi qu'il en soit, à travers ce panorama, c'est toute une nouvelle socio-économie qui se profile, où villages et industries semblent se conjuguer pour introduire des conditions particulières de croissance dont profitent sans doute bien des ménages, mais qui pourront aussi évoluer avec le temps. Le village de Saint-Eustache en offre un exemple. Prospère jusqu'à la fin des années 1830 , il perd par la suite plusieurs de ses anciennes fonctions, victime à la fois de la rébellion de 1837 et des concurrences de la ville et des bourgs voisins, et des bourgs situés dans l'arrière-pays seigneurial où prolifêre de plus en plus l'industrie rurale. De bourg industriel qu'il était encore en 1830, ce village se transforme progressivement en centre de services, où les fonctions commerciales et industrielles demeurent, mais sans l'ampleur qu'elles avaient auparavant. En 1831, ce bourg compte 832 habitants (500 en 1815). En 1851 , soit 14 ans après les troubles, il n'en compte plus que 783 , alors

\footnotetext{
48 Au recensement de 1831 , seul l'âge des hommes est ventilé par classe, celui des femmes ne l'étant qu'en fonction de la nubilité.

49 C'est le cas, entre autres, sur l'île de Montréal, où la proportion d'hommes mariés de 18 à 25 ans est toujours plus élevée dans les bourgs que dans la campagne environnante. Voir Montréal en 1825 d'après le recensement fait par Jacques Viger et Louis Guy (Montréal, Publication du Groupe d'études Gen-Histo Inc., 1977), 162-163. Données aimablement transmises par Pierre Tousignant, du département d'histoire de l'Université de Montréal.

50 Sur le système de reproduction sociale au Québec et ses effets dans l'espace, voir Gérard Bouchard, «Les systèmes de transmission des avoirs familiaux et le cycle de la société rurale au Québec, du XVIIe au XXe siècle», Histoire sociale/Social History, 16,31 (1983): 35-60, et «Sur la reproduction familiale en milieu rural: systèmes ouverts et systèmes clos», Recherches sociographiques, 28,2-3 (1987): 229-251; voir aussi Louise Dechêne, Habitants et marchands de Montréal au XVIIe siècle (Paris, Plon, 1974), 588 p.; Jacques Mathieu et al., «Peuplement colonisateur au XVIIIe siècle dans le Gouvernement de Québec», L'homme et la nature. Actes de la Société canadienne d'étude du XVIIIe siècle (Montréal, Société canadienne d'étude du XVIIIe siècle, 1984), 127-138, et «Mobilité et sédentarité: stratégies familiales en Nouvelle-France», Recherches sociographiques, 28,2-3 (1987): 211-227; et Jacques Mathieu et Serge Courville, dir., Peuplement colonisateur aux XVIIe et XVIIIe siècles (Québec, CELAT, coll. «Cahiers du CELAT», no 8, 1987), 292 p.
} 
que celui de Saint-Jérôme, par exemple, passe de 80 habitants environ à plus de 445 entre les deux dates. Avantageusement situé sur les rives de la rivière du Nord, au contact de la plaine et du plateau laurentien, ce dernier doit son origine à l'initiative des seigneurs Dumont et De Bellefeuille de Saint-Eustache, qui en planifient l'implantation dès les années $1830^{51}$. D'abord site d'une petite filature, il deviendra avec le temps l'un des principaux pôles industriels de la région nord de Montréal, dominant tout un réseau de moulins établis de part et d'autres de la rivière du Nord ${ }^{52}$.

Ce cas de Saint-Eustache n'a rien d'unique, il est celui de tous les bourgs qui ont eu ainsi à souffrir de l'apparition de nouveaux sites industriels mieux situés ou plus avantagés sur le plan des ressources. Celui de l'Assomption en offre un autre exemple, victime celui-là de la croissance du village d'Industrie où le seigneur Barthélemy Joliette avait établi autrefois des moulins. Jusqu'à l'agriculture qui en subira les effets: privée de ses débouchés traditionnels, elle connaîtra une longue phase d'hésitations, avant de s'orienter finalement dans l'élevage laitier et la production fourragère à destination du marché citadin et du marché extérieur. Et il n'y a pas que sur la rive nord que ce phénomène se produit. On l'observe également sur la rive sud du fleuve où, après les années 1840-1850, l'essor industriel avantage des bourgs bien identifiés (Saint-Hyacinthe, Saint-Jean, Valleyfield, etc.). Mais c'est peutêtre dans la vallée du Richelieu que les changements furent les plus notables, l'économie du blé faisant place ici à une économie laitière et de culture du foin comptant parmi les plus rentables de la région, stimulée, comme l'était autrefois le blé, par la demande sur le marché urbain et le marché extérieur, et reposant pour sa croissance sur les mêmes principes d'exclusion des enfants au moment du mariage, au profit d'un ou de quelques-uns. Parmi ces exclus, certains iront s'établir ailleurs sur une ferme. Les autres, plus nombreux, prendront le chemin de la ville ou des États-Unis voisins, où ils iront grossir les rangs du prolétariat urbain. En 1851, celle de Montréal compte quelque 58000 habitants. Dix ans plus tard, elle en comptera près de 90000 , environ 100000 avec sa banlieue, dont plusieurs ont une origine régionale ${ }^{53}$.

51 Un plan de ce village est disponible aux Archives du diocèse de Saint-Jérôme, sous la cote 332.101 H, 831-1. Intitulé «Dumontville», il est joint à une lettre du seigneur Dumont à $M$. Tabeau, vicaire général, datée du 14 janvier 1834. Il précise la morphologie du village, l'emplacement de l'église, etc., et prévoit même une rue bordée d'arbres...

52 Ce réseau a d'ailleurs été cartographié. Voir William Malsburg, «Plan showing different water powers of the Rivière du Nord at and near the village of St. Jerome county of Terrebonne, P.Q.», ler février 1871 . Ce plan, tracé à l'échelle de 360 pieds au pouce, montre la répartition des lots de moulins de part et d'autre du village de Saint-Jérôme. Il est conservé aux Archives du Séminaire de Québec, V 103, tiroir 215, no 16, Terrebonne, Saint-Jérôme.

53 Voir Jean-Claude Robert, «Urbanisation et population: le cas de Montréal en 1861», Revue d'histoire de l'Amérique française, 35,4 (mars 1982): 523-535. En 1861, environ 9,7\% de la population de Montréal provient de la campagne. L'échantillon observé (la population immigrante de deux quartiers, soit 3281 individus répartis en 1105 ménages) montre que près de $18 \%$ 


\section{CONCLUSION}

Vue pendant longtemps comme une activité routinière, mal articulée au marché, devenue avec le temps une zone refuge pour la collectivité canadienne-française, puis comme une activité primitive mais rationnelle, plus sensible au marché mais sujette elle aussi à des malaises qui en compromettent la croissance, l'agriculture au Bas-Canada présente des traits qui, tout en trouvant diverses correspondances empiriques avec ces images globalisantes, révèlent des situations beaucoup plus diverses que celles entrevues jusqu'ici, notamment dans la plaine de Montréal où ses organisations paraissent très contrastées et surtout très influencées par les éléments du contexte environnant. C'est du moins ce qui ressort de la cartographie des données du recensement de 1831 , une fois défalquées du total les déclarations des chefs de ménage qui ne pratiquent pas d'agriculture. Cette cartographie montre qu'il existe également ce que nous avons appelé un marché des subsistances qui, en 1831 du moins, semble très actif et surtout très capable d'influences sur l'agriculture, en plus de lui procurer des revenus substantiels.

Le trait dominant de la plaine de Montréal à l'époque réside dans ses contrastes. D'un côté, il y a d'abord ceux qui opposent la rive nord et la rive sud du fleuve, puis, à l'intérieur de ces vastes sous-ensembles, ceux qui séparent les milieux insulaires et les secteurs riverains du fleuve des localités de l'intérieur ou de l'arrière-pays seigneurial, et enfin, ceux qui distinguent les vallées et les petites vallées-plaines des côteaux voisins. De l'autre, il y a les écarts qui séparent les exploitations entre elles: certaines, très petites, ne sont déjà plus à ranger parmi les exploitations agricoles, d'autres, plus grandes, visent de toute évidence la production de surplus réguliers pour le marché, alors que d'autres, sont dans une situation intermédiaire d'activité qui, tout en restant sensible aux échanges, vise surtout la subsistance du ménage.

S'il faut y voir une conséquence de la diversité écologique introduite par un milieu physique lui-même très contrasté, il faut y voir aussi le résultat de conditions démographiques, économiques et sociales très diverses. Dans l'archipel de Montréal, la «péninsule» Vaudreuil-Soulanges et sur la rive nord du fleuve, où les types de terrain, l'hydrologie, le voisinage urbain, les densités de population, de bourgs et d'industries rurales incitent à une plus grande diversité dans les productions, l'agriculture s'oriente vers une polyculture céréalière et un élevage destiné surtout à la satisfaction de la demande intérieure. Sur la rive sud du fleuve, où les terres neuves sont plus abondantes, les surfaces argileuses

des migrants proviennent de la rive sud du fleuve, dont $10 \%$ du Richelieu. Toutefois, les plus forts contingents $(41,9 \%)$ proviennent de la rive nord, où la presque totalité des paroisses comprises entre l'Outaouais et Berthier sont représentées dans les listes. Quant à ceux qui proviennent de l'île de Montréal, elle n'est que de $9 \%$ environ. Communication personnelle de l'auteur. 
plus étendues, les voies de pénétration plus larges, le réseau routier plus dense et l'économie du blé bien structurée, elle est plus tournée vers le marché extérieur. Localement, les écarts sont plus grands encore, suggérant une activité d'autant plus spéculative que l'on se rapproche des cours d'eau et des secteurs riches en villages et en industries rurales.

Loin de créer des oppositions à l'intérieur de la région, ces contrastes sont des facteurs d'échanges importants entre les différentes parties du territoire, certains secteurs géographiques devenant des réservoirs de main-d'oeuvre pour d'autres plus industrialisées ou d'agriculture plus commerciale, d'autres, des zones actives de commercialisation des produits de l'intérieur, et d'autres encore, des lieux d'emplois variés pour ceux que l'agriculture n'attire plus ou qui en furent exclus. Ils sont aussi des facteurs importants de consolidation de l'agriculture locale qui, selon sa position dans l'espace et l'évolution de la demande dans le temps, pourra s'orienter vers différents types de marché, l'un suppléant à l'autre le cas échéant. Car même dans les zones de grandes cultures céréalières destinées au marché extérieur, on observe des combinaisons agricoles dont l'explication réside dans les orientations de la demande sur le marché local.

Cet équilibre semble définir un contexte très semblable à ceux observés dans d'autres régions du monde, où marché et industrie se conjuguent pour offrir à l'agriculture de nouveaux débouchés dont profitent plusieurs agriculteurs, pendant qu'à côté se multiplie le nombre de ceux pour qui elle ne devient plus qu'une activité secondaire dans laquelle on investit peu. Pourtant, comme tout équilibre, celui-ci est fragile, compromis bientôt par toute une série de changements préparés de longue date, mais qui ne se manifestent vraiment qu'après les années 1830 , alors que la concurrence des industries urbaines (et péri-urbaines) s'accentue et que l'extension des fronts pionniers entraîne le déplacement des pôles traditionnels de croissance vers d'autres secteurs plus avantagés sur le plan des ressources ou de l'accès au marché. Ajoutés aux accidents climatiques et épidémiologiques de la période, au manque réel ou spéculatif de terres et à l'attrait qu'exercent de plus en plus la ville et le travail salarié sur les masses paysannes, ces facteurs deviennent bientôt déterminants dans la montée des malaises ruraux. Ressentis par les cultivateurs de métier, ces malaises le sont également par les journaliers et les artisans, dont plusieurs épousent la cause patriote $^{54}$. Après 1840 , cette évolution s'accélère. Des symbioses demeurent, mais qui s'inscrivent dans un contexte renouvelé. Suit une longue période d'exode rural à destination de la ville et des bourgs où

\footnotetext{
54 À ce sujet, voir Lucie Blanchette-Lessard et Nicole Daigneault-Saint-Denis, «La participation des groupes sociaux aux rébellions dans les comtés de Laprairie et de Deux-Montagnes», Jean-Paul Bernard, dir., Les Rébellions de 1837-1838 (Montréal, Boréal Express, 1983), 327337.
} 
se concentre désormais l'industrie. L'agriculture entre alors dans une nouvelle phase de croissance marquée par l'agrandissement de la propriété foncière et bientôt le machinisme. Quant au développement industriel lui-même, il prendra d'autres voies. Toutefois, considérant la place que continueront d'occuper les petites et les moyennes entreprises dans le panorama économique du Québec, on peut se demander si la phase préalable d'industrialisation enregistrée à la campagne n'a pas préparé finalement la montée de l'âge industriel, ce qui nous amènerait à revoir d'un autre oeil l'histoire économique et sociale du BasCanada. 